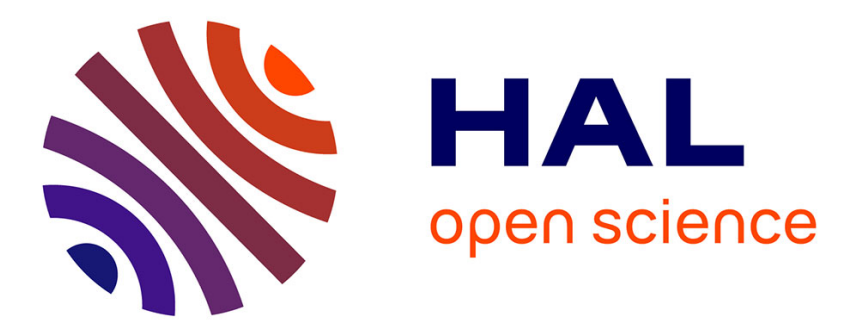

\title{
Averaging of a stochastic slow-fast model for population dynamics: application to the development of ovarian follicles
}

Guillaume Ballif, Frédérique Clément, Romain Yvinec

\section{- To cite this version: \\ Guillaume Ballif, Frédérique Clément, Romain Yvinec. Averaging of a stochastic slow-fast model for population dynamics: application to the development of ovarian follicles. SIAM Journal on Applied Mathematics, 2022, 82 (1), pp.359-380. 10.1137/21M1409615 . hal-03405177}

\author{
HAL Id: hal-03405177 \\ https://hal.science/hal-03405177
}

Submitted on 27 Oct 2021

HAL is a multi-disciplinary open access archive for the deposit and dissemination of scientific research documents, whether they are published or not. The documents may come from teaching and research institutions in France or abroad, or from public or private research centers.
L'archive ouverte pluridisciplinaire $\mathbf{H A L}$, est destinée au dépôt et à la diffusion de documents scientifiques de niveau recherche, publiés ou non, émanant des établissements d'enseignement et de recherche français ou étrangers, des laboratoires publics ou privés. 


\title{
AVERAGING OF A STOCHASTIC SLOW-FAST MODEL FOR POPULATION DYNAMICS: APPLICATION TO THE DEVELOPMENT OF OVARIAN FOLLICLES*
}

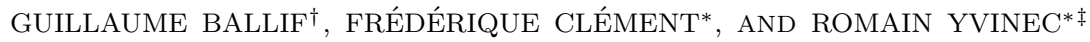

\begin{abstract}
We analyze a birth, migration and death stochastic process modeling the dynamics of a finite population, in which individuals transit unidirectionally across successive compartments. The model is formulated as a continuous-time Markov chain, whose transition matrix involves multiscale effects; the whole (or part of the) population affects the rates of individual birth, migration and death events. Using the slow-fast property of the model, we prove the existence and uniqueness of the limit model in the framework of stochastic singular perturbations. The derivation of the limit model is based on compactness and coupling arguments. The uniqueness is handled by applying the ergodicity theory and studying a dedicated Poisson equation. The limit model consists of an ordinary-differential equation ruling the dynamics of the first (slow) compartment, coupled with a quasi-stationary distribution in the remaining (fast) compartments, which averages the contribution of the fast component of the Markov chain on the slow one. We illustrate numerically the convergence, and highlight the relevance of dealing with nonlinear event rates for our application in reproductive biology. The numerical simulations involve a simple integration scheme for the deterministic part, coupled with the nested algorithm to sample the quasi-stationary distribution.
\end{abstract}

Key words. Continuous time Markov chain - Singular perturbations - Stochastic coupling techniques - Foster-Lyapunov criterion - Nested algorithm - Reproductive biology

AMS subject classifications. 92D25, 60G10, 60J28.

1. Introduction. We study a birth, migration and death stochastic process modeling the dynamics of a finite population, in which individuals transit unidirectionally across successive compartments. The model is formulated as a continuous-time Markov chain, whose transition matrix involves multiscale effects ; the whole (or part of the) population affects the rates of individual birth, migration and death events. The model is characterized by two contrasted time and abundance scales: the first compartment has a high abundance with a slow dynamics, and the remaining compartments have order one abundances with fast dynamics.

The first formulation of the model, as a death and migration process without birth, was introduced in [1] to follow the changes in the population of ovarian follicles throughout lifespan. Ovarian follicles are dynamic endocrine units of the ovaries, enclosing a single germ cell. Each follicle undergoes a sequence of developmental stages, starting with activation from an initially quiescent state, and ending with either ovulation from the final stage or (most of the time) degeneration at any stage. This model is especially suited for mammals, in which a numerous pool of quiescent follicles (the reserve) is constituted once for all in the pre- or peri-natal period. As soon as follicles are formed, and all along life until the pool is exhausted, follicles get slowly activated in an asynchronous way and progress more rapidly through different maturation steps. Hence, at any time, the ovarian function can be characterized by the total number of follicles, and in particular of remaining quiescent follicles, and by the distribution of growing follicles according to their developmental stage. This distribution is further shaped by the control exerted onto the growth (migration) and death rates by reproductive hormones, which emanate (either directly or indirectly)

\footnotetext{
* Submitted to the editors on April 2, 2021.

†Université Paris-Saclay, Inria, Inria Saclay-Île-de-France, 91120, Palaiseau, France (guillaume.ballif@inria.fr, frederique.clement@inria.fr)

${ }^{\ddagger}$ PRC, INRAE, CNRS, Université de Tours, 37380 Nouzilly, France (romain.yvinec@inria.fr)
} 
from the follicles themselves (see [2] and references therein). Such controls notably prevent the early exhaustion of the quiescent pool, and are at the source of the cyclic activity of the ovaries. Accordingly, and in contrast to former simpler, linear models [8], the nonlinear expressions for the transition rates include information on follicle numbers in each or all stages. The shape of the follicle distribution is remarkably similar between different mammalian species and with time (except at the very beginning and very end of follicle development, when some compartments are still not filled, or on the contrary, have been exhausted). This distribution both results from and participates in the endocrine homeostasis of the ovaries, and more generally of the reproductive (hypothalamic-pituitary-gonadal) axis. It also ensures that a proper number of mature germ cells is supplied each ovarian cycle for ovulation during all reproductive life.

In [1], taking advantage of the timescale difference between follicle activation and follicle growth, the authors conjectured the existence of a reduced deterministic limit model. In the current study, we derive rigorously the reduction on an extended model including birth events in the reserve. The birth events enable the quiescent pool to be renewed, as encountered in several zoological classes (fishes for instance). We also illustrate numerically the convergence of the full model towards the limit model, as the scaling parameter decreases to zero, and we further explore the behavior of the limit model to highlight the salient features of the nonlinear formulation of the model. The limit model consists of an ordinary differential equation ruling the dynamics of the first compartment, coupled with a quasi-stationary distribution in the remaining compartments, which averages the contribution of the fast component of the Markov chain on the slow one. To derive the limit model, we make use of the generic framework of averaging, suitable to handle singular perturbation problems [14]. Such reduction techniques have found more and more applications in biology, in particular in chemical reaction networks or population dynamics [13]. The main ingredients needed to identify and prove the limit behavior rely on compactness arguments, that we handle here thanks to a Foster-Lyapunov criterion [16], and on coupling arguments [15]. An additional crucial step, rarely addressed in similar studies up to our knowledge, is the uniqueness of the solution of the limit model, which we handle by means of the ergodicity theory and Poisson equation, a classical tool in sensitivity analysis [5].

In the linear case (with constant, uncontrolled event rates), direct biologically relevant information can be extracted from the analytical expression of the limit model, as the speed of exhaustion of the first compartment or the shape of the follicle distribution and its sensitivity to parameters. This is not the case in the nonlinear case, for which we settled an appropriate numerical strategy to capture both the dynamics of the slow compartment and the quasi-stationary distribution of the fast compartments. We have combined a simple Euler scheme, to follow the dynamics of the deterministic part, with the nested algorithm [23], to sample efficiently the quasi-stationary distribution of the fast part of the Markov chain. This numerical framework allows us to illustrate the convergence of the initial model towards the limit model, as well as the effects of nonlinear event rates on the model behavior.

The manuscript is organized as follows. The model and the main result are presented in section 2, together with a detailed outline of the main steps for the proof of the main result. Compactness and ergodicity properties through the FosterLyapunov criterion are given in section 3. Section 4 is dedicated to the proof of the main result using the averaging tools. In section 5, we illustrate numerically the convergence result, using a nested algorithm to simulate the limit model, and we develop a specific application in reproductive biology. Finally, we discuss our results 
in section 6.

\section{Model presentation and main results.}

2.1. Introduction of the model. We consider a population of $X$ quiescent individuals in a slow compartment (the reserve), and a population of individuals distributed into successive fast compartments with increasing maturity $Y=\left(Y_{i}\right)_{i \in \llbracket 1 ; d \rrbracket}$. Individuals may leave the reserve to enter the first fast compartment $Y_{1}$, at rate $\lambda_{0}$, or die at rate $\mu_{0}$. In each compartment $i$, individuals may either progress to the next compartment $i+1$, at rate $\lambda_{i}$ (except in the last compartment), or die at rate $\mu_{i}$. In addition, we complete the initial formulation of [1] by adding a birth term, at rate $r_{0}$ in the slow compartment $X$. After introducing a small parameter $\varepsilon$, rescaling time from $t$ to $\frac{t}{\varepsilon}$ and the reserve size from $X$ to $\varepsilon X$, and multiplying by $\varepsilon$ the rates $\lambda_{0}, \mu_{0}$ and $r_{0}$, the model can be fully characterized by the following state transitions:

\begin{tabular}{|c|c|c|}
\hline & Transition & Rate \\
\hline Birth (reserve) & $\left(X^{\varepsilon}, Y^{\varepsilon}\right) \rightarrow\left(X^{\varepsilon}+\varepsilon, Y^{\varepsilon}\right)$ & $\frac{r_{0}\left(Y^{\varepsilon}\right)}{\varepsilon} X^{\varepsilon}$ \\
\hline Maturation (reserve) & $\left(X^{\varepsilon}, Y^{\varepsilon}\right) \rightarrow\left(X^{\varepsilon}-\varepsilon, Y^{\varepsilon}+e_{1}\right)$ & $\frac{\lambda_{0}\left(Y^{\varepsilon}\right)}{\varepsilon} X^{\varepsilon}$ \\
\hline Death (reserve) & $\left(X^{\varepsilon}, Y^{\varepsilon}\right) \rightarrow\left(X^{\varepsilon}-\varepsilon, Y^{\varepsilon}\right)$ & $\frac{\mu_{0}\left(Y^{\varepsilon}\right)}{\varepsilon} X^{\varepsilon}$ \\
\hline Maturation, $i \in \llbracket 1, d-1 \rrbracket$ & $\left(X^{\varepsilon}, Y^{\varepsilon}\right) \rightarrow\left(X^{\varepsilon}, Y^{\varepsilon}-e_{i}+e_{i+1}\right)$ & $\frac{\lambda_{i}\left(Y^{\varepsilon}\right)}{\varepsilon} Y_{i}^{\varepsilon}$ \\
\hline Death, $i \in \llbracket 1, d \rrbracket$ & $\left(X^{\varepsilon}, Y^{\varepsilon}\right) \rightarrow\left(X^{\varepsilon}, Y^{\varepsilon}-e_{i}\right)$ & $\frac{\mu_{i}\left(Y^{\varepsilon}\right)}{\varepsilon} Y_{i}^{\varepsilon}$ \\
\hline
\end{tabular}

TABLE 1

Transitions of the process $\left(X^{\varepsilon}(t), Y^{\varepsilon}(t)\right)_{t \geq 0}$ in $\mathbb{R}_{+} \times \mathbb{N}^{d}$. We denote by $e=\left(e_{i}\right)_{i \in \llbracket 1 ; d \rrbracket}$ the canonical basis of $\mathbb{N}^{d}$. To simplify some computations, we set $\lambda_{d} \equiv 0$.

2.2. Hypotheses. To ensure the existence of the process defined in Table 1 at all times, we make the following hypotheses on the rates of events affecting the reserve :

Hypothesis 2.1 (Birth rate $r_{0}$ and migration rate $\lambda_{0}$ ).

$$
\begin{aligned}
& \exists R_{0}>0, \forall y \in \mathbb{N}^{d}, r_{0}(y) \leq R_{0}, \\
& \exists B_{0}>0, \quad \forall y \in \mathbb{N}^{d}, \lambda_{0}(y) \leq B_{0} .
\end{aligned}
$$

The existence of the stationary probability distribution is strongly linked to the nonaccumulation of individuals in a compartment. Accordingly, we assume that

Hypothesis 2.2 (Migration rates $\lambda_{i}$ ).

$$
\forall i \in \llbracket 0, d-1 \rrbracket, \forall y \in \mathbb{N}^{d}, \lambda_{i}(y)>0,
$$

Hypothesis 2.3 (Non-accumulation).

$$
\forall i \in \llbracket 0, d \rrbracket, \alpha_{i}:=\inf _{y \in \mathbb{N}^{d}}\left(\lambda_{i}(y)+\mu_{i}(y)\right)>0 .
$$


We also make a technical assumption on all event rates :

Hypothesis 2.4 (Polynomial rates).

$\exists m_{0} \in \mathbb{N}, \exists D_{0}>0, \forall y \in \mathbb{N}^{d},\left\{\begin{array}{ll}\forall i \in \llbracket 1, d-1 \rrbracket, & \lambda_{i}(y) \leq D_{0}\left[1+\left(\sum_{j=1}^{d} y_{j}\right)^{m_{0}}\right] \\ \forall i \in \llbracket 0, d \rrbracket, & \mu_{i}(y) \leq D_{0}\left[1+\left(\sum_{j=1}^{d} y_{j}\right)^{m_{0}}\right] .\end{array}\right.$.

2.3. Notations and definitions. We use the following standard notations :

- $\mathcal{C}_{b}\left(\mathbb{N}^{d}\right)=\left\{f: \mathbb{N}^{d} \rightarrow \mathbb{R}\right.$ bounded $\}$,

- $\mathcal{D}_{\mathbb{N}^{d}}\left[0, \infty\left[\right.\right.$ : space of $\mathbb{N}^{d}$-valued functions that are right-continuous and leftlimited (càdlàg) on $[0, \infty[$,

- $\mathcal{L}_{m}\left(\mathbb{N}^{d}\right)$ : space of measure $\mu$ on $\left[0, \infty\left[\times \mathbb{N}^{d}\right.\right.$ such that $\mu\left(\left[0, t\left[\times \mathbb{N}^{d}\right)=t\right.\right.$,

- For $x \in \mathbb{R},(x)^{+}=\max (0, x)$,

- For $\nu$ a measure on $\mathbb{N}^{d}$ and $f: \mathbb{N}^{d} \mapsto \mathbb{R}$ a function integrable against $\nu$, $\langle\nu, f\rangle:=\sum_{x \in \mathbb{N}^{d}} f(x) \nu(x)$.

Also, the following notation will be used repeatedly:

- we define the following sets :

$$
\left\{\begin{array}{l}
\mathcal{P}=\left\{p \in\left(\mathbb{N}^{*}\right)^{d}, \forall i \in \llbracket 1, d-1 \rrbracket, p_{i}>p_{i+1},\right\} \\
\mathcal{S}=\left\{w \in \mathbb{N}^{d}, \forall i \in \llbracket 1, d-1 \rrbracket, w_{i} \leq w_{i+1}\right\}
\end{array}\right.
$$

- For $Z$ a d-dimensional process in $\mathcal{D}_{\mathbb{N}^{d}}\left[0, \infty\left[, Z_{\text {tot }}\right.\right.$ denotes $\sum_{i=1}^{d} Z_{i}$.

Let us define rigorously the birth, death and migration process for any $\varepsilon>0$ :

Definition 2.5. Let $\left(X^{\varepsilon}(t), Y^{\varepsilon}(t)\right)_{t \geq 0}$ be the càdlàg d-dimensional Markov process defined in law by the following generator, for any $g: \mathbb{N}^{d} \times \mathbb{N} \mapsto \mathbb{R}$ bounded,

$$
\begin{aligned}
A^{\varepsilon} g(x, y) & =\frac{r_{0}(y)}{\varepsilon} x[g(x+\varepsilon, y)-g(x, y)]+\frac{\lambda_{0}(y)}{\varepsilon} x\left[g\left(x-\varepsilon, y+e_{1}\right)-g(x, y)\right] \\
& +\frac{\mu_{0}(y)}{\varepsilon} x[g(x-\varepsilon, y)-g(x, y)]+\sum_{i=1}^{d} \frac{\mu_{i}(y)}{\varepsilon} y_{i}\left[g\left(x, y-e_{i}\right)-g(x, y)\right] \\
(2.1) \quad & +\sum_{i=1}^{d-1} \frac{\lambda_{i}(y)}{\varepsilon} y_{i}\left[g\left(x, y+e_{i+1}-e_{i}\right)-g(x, y)\right] .
\end{aligned}
$$

Let $\left(\mathcal{F}_{t}^{\varepsilon}\right)_{t \geq 0}$ be the canonical filtration associated with $\left(X^{\varepsilon}(t), Y^{\varepsilon}(t)\right)_{t \geq 0}$.

We can now state our main result, the existence and uniqueness of the limit model obtained in the limit $\varepsilon \rightarrow 0$ :

THEOREM 2.6. For any initial condition $\left(X^{\varepsilon}(0), Y^{\varepsilon}(0)\right) \in \varepsilon \mathbb{N} \times \mathbb{N}^{d}$ such that, for some deterministic $x^{\text {in }} \in \mathbb{R}^{+}$,

$$
\forall k>1, \quad \sup _{\varepsilon} \mathbb{E}\left(X^{\varepsilon}(0)^{k}\right)<\infty, \quad \sup _{\varepsilon} \mathbb{E}\left(Y_{\text {tot }}^{\varepsilon}(0)^{k}\right)<\infty, \quad X^{\varepsilon}(0) \underset{a . s}{\longrightarrow} x^{i n},
$$


the process $\left(X^{\varepsilon}, Y^{\varepsilon}\right)$ is relatively compact in $\mathcal{D}_{\mathbb{R}^{+}}\left[0, \infty\left[\times \mathcal{L}_{m}\left(\mathbb{N}^{d}\right)\right.\right.$, and has a unique limit process $(x, \pi) \in \mathcal{C}^{1}\left(\mathbb{R}^{+}\right) \times \mathcal{L}_{m}\left(\mathbb{N}^{d}\right)$ such that :

$$
\left\{\begin{array}{l}
\frac{\mathrm{d} x}{\mathrm{~d} t}(t)=\Lambda_{0}(x(t)) x(t), \quad t>0, \text { with } x(0)=x^{i n} \\
\Lambda_{0}(x(t))=\sum_{y \in \mathbb{N}^{d}}\left(r_{0}(y)-\lambda_{0}(y)-\mu_{0}(y)\right) \pi_{x(t)}(y)
\end{array}\right.
$$

where, for $x \in \mathbb{R}^{+}, \pi_{x}$ verifies

$$
\forall f \in \mathcal{C}_{b}\left(\mathbb{N}^{d}\right),\left\langle L_{x} f, \pi_{x}\right\rangle=0,
$$

with $L_{x}$ the following generator defined for any function $f \in \mathcal{C}_{b}\left(\mathbb{N}^{d}\right)$ :

$$
\begin{aligned}
\forall y \in \mathbb{N}^{d}, L_{x} f(y) & =\lambda_{0}(y) x\left[f\left(y+e_{1}\right)-f(y)\right]+\sum_{i=1}^{d-1} \lambda_{i}(y) y_{i}\left[f\left(y+e_{i+1}-e_{i}\right)-f(y)\right] \\
(2.2) & +\sum_{i=1}^{d} \mu_{i}(y) y_{i}\left[f\left(y-e_{i}\right)-f(y)\right] .
\end{aligned}
$$

Remark 2.7 (Initial condition $x^{\text {in }}$ ). Theorem 2.6 remains true with $x^{\text {in }}$ a random variable with a finite expectation. The proofs remain the same, but are lighter with a deterministic initial condition.

The proof of Theorem 2.6 is divided in two parts, exposed in sections 3 and 4 . The main strategy is to use the tools developed in [14], which adapt the standard compactness/identification of the limit scheme to stochastic singular perturbation problems. The compactness of the slow component $X^{\varepsilon}$ is obtained thanks to standard criteria on $\mathcal{D}_{\mathbb{R}^{+}}\left[0, \infty\left[\right.\right.$. The fast component $Y^{\varepsilon}$ does not converge in a functional sense, but its occupation measure does, which leads to the study of a (quasi-)stationary problem associated with the generator defined in (2.2). We deploy this machinery in section 4, with compactness criteria in subsection 4.1 and identification of the limit in subsection 4.2. Both suitable moment control on $\left(X^{\varepsilon}, Y^{\varepsilon}\right)$ and ergodicity associated with the generator are proved in section 3, using a relevant change of variables. Looking at the cumulative sums associated with $Y^{\varepsilon}$ rather than at the individual numbers in each compartment allows one to exhibit a linear stochastic process that dominates $\left(X^{\varepsilon}, Y^{\varepsilon}\right)$ (by coupling methods), and to obtain a uniform moment control on $\left(X^{\varepsilon}, Y^{\varepsilon}\right)$ (Proposition 3.1). Similarly, rewriting the generator in terms of cumulative sums allows one to prove the irreducibility, and to find an appropriate Lyapounov function enabling to deduce the existence and uniqueness of $\pi_{x}$ with minimal assumptions, as well as the local Lipschitz-continuity in $x$ of integrals against $\pi_{x}$ (Theorem 3.6).

\section{Control and ergodicity of the process.}

\subsection{Uniform control of the moments of $X^{\varepsilon}$ and $Y^{\varepsilon}$.}

Proposition 3.1. Let $\left(X^{\varepsilon}(0), Y^{\varepsilon}(0)\right) \in \varepsilon \mathbb{N} \times \mathbb{N}^{d}$ be an initial condition for the process. With the same assumptions as in Theorem 2.6, we have:

$$
\begin{array}{r}
\forall k>1, \forall T>0, \sup _{\varepsilon} \mathbb{E}\left(\sup _{t \leq T}\left|X^{\varepsilon}(t)\right|^{k}\right)<\infty, \\
\forall k>1, \sup _{\varepsilon} \sup _{t \geq 0} \mathbb{E}\left(\left|Y_{\text {tot }}^{\varepsilon}(t)\right|^{k}\right)<\infty .
\end{array}
$$


To prove Proposition 3.1, we introduce in Definition 3.2 a stochastic process $\left(M^{\varepsilon}, N^{\varepsilon}\right)$ whose transition rates are linear with respect to the state variables. Writing the fast component in terms of cumulative sums, and coupling $\left(X^{\varepsilon}, Y^{\varepsilon}\right)$ with the linear process $\left(M^{\varepsilon}, N^{\varepsilon}\right)$, we can show that $\left(M^{\varepsilon}, N^{\varepsilon}\right)$ is a majorizing process for $\left(X^{\varepsilon}, Y^{\varepsilon}\right)$. The moments of $\left(M^{\varepsilon}, N^{\varepsilon}\right)$ can then be controlled thanks to a Lyapounov function (Lemma 3.4).

Definition 3.2. Let $\left(M^{\varepsilon}(t), N^{\varepsilon}(t)\right)_{t \geq 0}$ be the càdlàg d-dimensional Markov process defined in law by the following generator $B^{\varepsilon}$, for any function $g \in \mathcal{C}_{b}\left(\mathbb{R}^{+} \times \mathbb{N}^{d}\right)$ :

$B^{\varepsilon} g(m, n)=\frac{R_{0}}{\varepsilon} m[g(m+\varepsilon, n)-g(m, n)]+\frac{B_{0}}{\varepsilon} m\left[g\left(m, n+e_{1}\right)-g(m, n)\right]$

$$
+\frac{\alpha_{0}}{\varepsilon} m\left[g\left(m-\varepsilon, n+e_{1}\right)-g(m, n)\right]+\sum_{i=1}^{d} \frac{\alpha_{i}}{\varepsilon} n_{i}\left[g\left(m, n+e_{i+1}-e_{i}\right)-g(m, n)\right] .
$$

We introduce the following change of variables for $1 \leq i \leq d$,

$$
V_{i}^{\varepsilon}(t)=\sum_{j=1}^{i} Y_{j}^{\varepsilon}(t), \quad W_{i}^{\varepsilon}(t)=\sum_{j=1}^{i} N_{j}^{\varepsilon}(t)
$$

It is clear that $V^{\varepsilon}, W^{\varepsilon} \in \mathcal{S}$. With a slight abuse of notation, the generators $A^{\varepsilon}$ (2.1) and $B^{\varepsilon}(3.1)$ become for any $v, w \in \mathcal{S}$ (with $v_{0}=w_{0}=0$ ):

$$
\begin{aligned}
A^{\varepsilon} g(x, v) & =\frac{r_{0}(v)}{\varepsilon} x[g(x+\varepsilon, v)-g(x, v)]+\frac{\lambda_{0}(v)}{\varepsilon} x\left[g\left(x-\varepsilon, v+\sum_{j=1}^{d} e_{j}\right)-g(x, v)\right] \\
& +\frac{\mu_{0}(v)}{\varepsilon} x[g(x-\varepsilon, v)-g(x, v)] \\
& +\sum_{i=1}^{d} \frac{\mu_{i}(v)}{\varepsilon}\left(v_{i}-v_{i-1}\right)\left[g\left(x, v-\sum_{j=i}^{d} e_{j}\right)-g(x, v)\right] \\
& +\sum_{i=1}^{d-1} \frac{\lambda_{i}(v)}{\varepsilon}\left(v_{i}-v_{i-1}\right)\left[g\left(x, v-e_{i}\right)-g(x, v)\right], \\
B^{\varepsilon} g(m, w) & =\frac{R_{0}}{\varepsilon} m[g(m+\varepsilon, w)-g(m, w)]+\frac{B_{0}}{\varepsilon} m\left[g\left(m, w+\sum_{i=1}^{d} e_{i}\right)-g(m, w)\right] \\
& +\frac{\alpha_{0}}{\varepsilon} m\left[g\left(m-\varepsilon, w+\sum_{i=1}^{d} e_{i}\right)-g(m, w)\right] \\
& +\sum_{j=1}^{d} \frac{\alpha_{i}}{\varepsilon}\left(w_{i}-w_{i-1}\right)\left[g\left(m, w-e_{i}\right)-g(m, w)\right] .
\end{aligned}
$$

We prove in the next lemma that $\left(X^{\varepsilon}, V^{\varepsilon}\right)$ is dominated by $\left(M^{\varepsilon}, W^{\varepsilon}\right)$.

LEMmA 3.3. For any $\left(M^{\varepsilon}(0), N^{\varepsilon}(0)\right)$ such that $X^{\varepsilon}(0) \leq M^{\varepsilon}(0)$ and $Y^{\varepsilon}(0) \leq$ 
$N^{\varepsilon}(0)$, we have, for any $t>0$,

$$
\left\{\begin{array}{l}
X^{\varepsilon}(t) \leq M^{\varepsilon}(t) \text { stochastically } \\
V^{\varepsilon}(t) \leq W^{\varepsilon}(t) \text { stochastically componentwise. }
\end{array}\right.
$$

Proof. We introduce a coupling between processes $\left(X^{\varepsilon}, V^{\varepsilon}\right)$ and $\left(M^{\varepsilon}, W^{\varepsilon}\right)$ with the following events driven by the slow components $\left(X^{\varepsilon}, M^{\varepsilon}\right)$ :

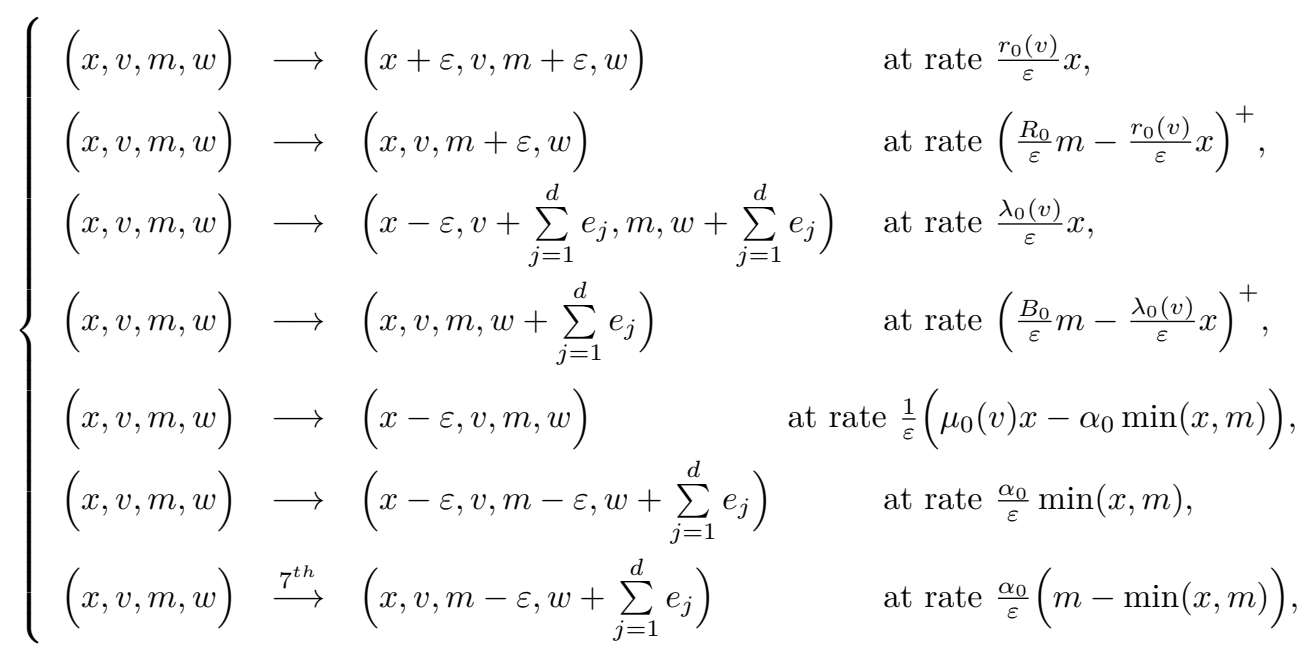

and the following events driven by the fast components $\left(V^{\varepsilon}, W^{\varepsilon}\right)$, for any $i \in \llbracket 1, d \rrbracket$ :

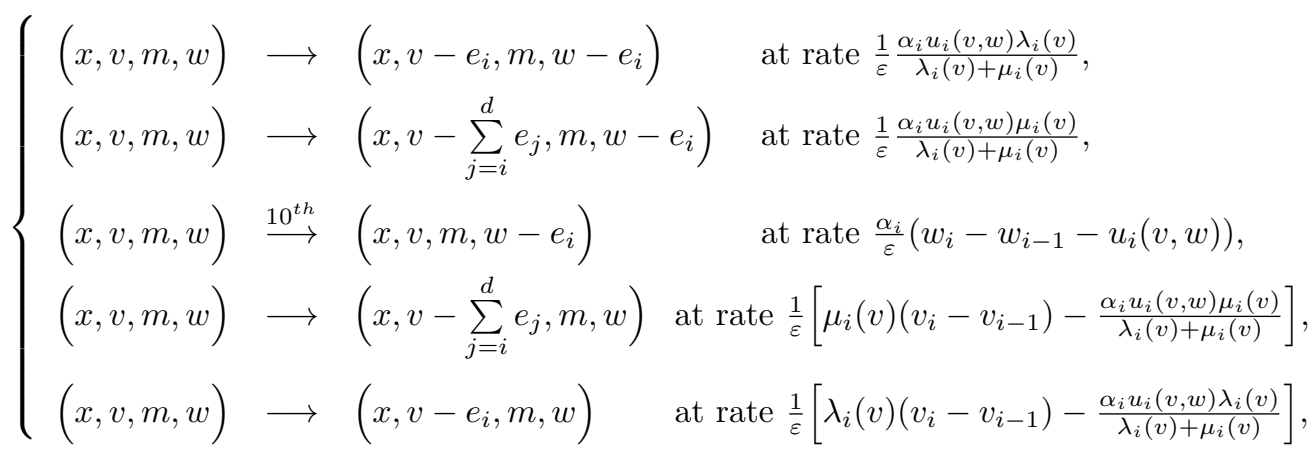

with $u_{i}(v, w)=\min \left(v_{i}-v_{i-1}, w_{i}-w_{i-1}\right)$.

The marginal $\left(X^{\varepsilon}, V^{\varepsilon}\right)$ has for generator $A^{\varepsilon}$ (3.3) and the marginal $\left(M^{\varepsilon}, W^{\varepsilon}\right)$ has for generator $B^{\varepsilon}$ (3.4), as long as $X^{\varepsilon} \leq M^{\varepsilon}$ (see section SM1). Furthermore, each event preserves the order $X^{\varepsilon} \leq M^{\varepsilon}$ except the seventh event. Since $X^{\varepsilon}, M^{\varepsilon} \in \varepsilon \mathbb{N}$ and the jump increment of the seventh event is $-\varepsilon$, the order between $X^{\varepsilon}$ and $M^{\varepsilon}$ can be reversed only when $X^{\varepsilon}=M^{\varepsilon}$, in which case the rate is zero. Thus, the order $X^{\varepsilon} \leq M^{\varepsilon}$ is preserved.

In the same way, each event preserves the (componentwise) order $V^{\varepsilon} \leq W^{\varepsilon}$ except the tenth event. For any $i \in \llbracket 1, d \rrbracket$, since $V_{i}^{\varepsilon}, W_{i}^{\varepsilon} \in \mathbb{N}^{d}$ and the jump increment of the tenth event is -1 , the order between $V_{i}^{\varepsilon}$ and $W_{i}^{\varepsilon}$ can be reversed only when $V_{i}^{\varepsilon}=W_{i}^{\varepsilon}$. 
However, in any state such that

$$
V_{i}^{\varepsilon}=W_{i}^{\varepsilon} \text {, and } \forall j \neq i, V_{j}^{\varepsilon} \leq W_{j}^{\varepsilon}
$$

the rate of the tenth event is equal to 0 , so that the order $V^{\varepsilon} \leq W^{\varepsilon}$ is preserved, which achieves the proof.

We now prove that the moments of $\left(M^{\varepsilon}, W^{\varepsilon}\right)$ are uniformly bounded.

LEMma 3.4. For any sequence of initial condition $\left(M^{\varepsilon}(0), W^{\varepsilon}(0)\right) \in \varepsilon \mathbb{N} \times \mathbb{N}^{d}$ such that

$$
\forall k>1, \sup _{\varepsilon} \mathbb{E}\left(M^{\varepsilon}(0)^{k}\right)<\infty, \sup _{\varepsilon} \mathbb{E}\left(W_{t o t}^{\varepsilon}(0)^{k}\right)<\infty,
$$

we have, for the slow component,

$$
\forall k>1, \forall T>0, \sup _{\varepsilon} \mathbb{E}\left(\sup _{t \leq T}\left|M^{\varepsilon}(t)\right|^{k}\right)<\infty
$$

and, for the fast component,

$$
\forall k>1, \sup _{\varepsilon} \sup _{t \geq 0} \mathbb{E}\left(\left|W_{t o t}^{\varepsilon}(t)\right|^{k}\right)<\infty .
$$

Proof. $M^{\varepsilon}$ is a linear birth and death process with birth (resp. death) rate $\frac{R_{0}}{\varepsilon} m$ (resp. $\frac{\alpha_{0}}{\varepsilon} m$ ). Since the jumps increment $M^{\varepsilon}$ by $\pm \varepsilon$, we obtain (3.5) by a straightforward adaptation of [17, Proposition 2.7].

Let $p \in \mathcal{P}$. We will prove that function $V_{p}$, defined for any $w \in \mathcal{S}$ by $V_{p}(w)=\sum_{i=1}^{d} w_{i}^{p_{i}}$, is a Lyapunov function for $W^{\varepsilon}$, that is, there exists a pair $a_{1}, a_{2}>0$ and a compact set $C \subset \mathbb{N}^{d}$ such that:

$$
B^{\varepsilon} V_{p}(m, w) \leq \frac{a_{2}}{\varepsilon} \mathbb{1}_{C}(w)-\frac{a_{1}}{\varepsilon} V_{p}(w)
$$

From (3.4) and using Lemma SM2.1, we obtain, for any $w \in \mathcal{S}$ :

$$
\begin{aligned}
B^{\varepsilon} V_{p}(m, w)= & \frac{B_{0}+\alpha_{0}}{\varepsilon} m \sum_{i=1}^{d}\left[\left(w_{i}+1\right)^{p_{i}}-w_{i}^{p_{i}}\right] \\
& +\sum_{i=1}^{d} \frac{\alpha_{i}}{\varepsilon}\left(w_{i}-w_{i-1}\right)\left[\left(w_{i}-1\right)^{p_{i}}-w_{i}^{p_{i}}\right] \\
\leq & \frac{B_{0}+\alpha_{0}}{\varepsilon} m \sum_{i=1}^{d} 2^{p_{i}}\left(w_{i}^{p_{i}-1}+1\right)-\sum_{i=1}^{d} \frac{\alpha_{i}}{\varepsilon}\left(w_{i}-w_{i-1}\right) w_{i}^{p_{i}-1} \\
\leq & \frac{F(w)}{\varepsilon}-\frac{\alpha}{2 \varepsilon} V_{p}(w),
\end{aligned}
$$

where $\alpha=\min _{1 \leq i \leq d-1} \alpha_{i}>0$ from Hypothesis 2.3, and $F$ is given by, for $w \in \mathcal{S}$,

$$
F(w)=\left(B_{0}+\alpha_{0}\right) m \sum_{i=1}^{d} 2^{p_{i}}\left(w_{i}^{p_{i}-1}+1\right)+\alpha \sum_{i=1}^{d} w_{i-1} w_{i}^{p_{i}-1}-\frac{\alpha}{2} V_{p}(w) .
$$

To prove (3.7), we observe that, for any $i, w_{i} \leq V_{p}(w)^{1 / p_{i}}$, so that, for any $p \in \mathcal{P}$,

$$
\lim _{\|w\| \rightarrow \infty} \frac{F(w)}{V_{p}(w)}=-\frac{\alpha}{2} \text {. }
$$


Hence, there is a compact set $C$ such that $F$ is negative on $\mathbb{N}^{d} \backslash C$. In consequence, $F$ reaches a finite maximal value on $\mathbb{N}^{d}$, leading to (3.7) with $a_{1}=\alpha / 2$ and $a_{2}=$ $\max _{w \in \mathbb{N}^{d}} F(w)<\infty$. We now deduce from (3.7) that

$$
\begin{aligned}
\mathbb{E}\left[V_{p}\left(W^{\varepsilon}(t)\right)\right] \leq \exp \left(-\frac{a_{1}}{\varepsilon} t\right) \mathbb{E}\left[V_{p}\left(W^{\varepsilon}(0)\right)\right]+\frac{a_{2}}{a_{1}} & \left(1-\exp \left(-\frac{a_{1}}{\varepsilon} t\right)\right) \\
& \leq \mathbb{E}\left[V_{p}\left(W^{\varepsilon}(0)\right)\right]+\frac{a_{2}}{a_{1}}
\end{aligned}
$$

Since (3.9) is verified for all $p \in \mathcal{P}$, all $\varepsilon>0$ and all $t \geq 0$, we finally obtain (3.6).

Proof of Proposition 3.1. We apply Lemma 3.3 with Lemma 3.4, and use the inequality $Y_{\text {tot }}^{\varepsilon} \leq V_{\text {tot }}^{\varepsilon}$, to obtain Proposition 3.1.

3.2. Existence and uniqueness of an invariant probability measure. We introduce the process $Y$ which represents the fast part of $\left(X^{\varepsilon}, Y^{\varepsilon}\right)$, for a frozen value of $X^{\varepsilon}$. In all this subsection, let $x>0$ be a fixed positive number.

DeFinition 3.5. Let $Y$ be the càdlàg d-dimensional Markov process defined in law by the following generator, for any $f: \mathbb{N}^{d} \times \mathbb{N} \mapsto \mathbb{R}$ bounded,

$$
\begin{aligned}
\forall y \in \mathbb{N}^{d}, L_{x} f(y) & =\lambda_{0}(y) x\left[f\left(y+e_{1}\right)-f(y)\right]+\sum_{i=1}^{d-1} \lambda_{i}(y) y_{i}\left[f\left(y+e_{i+1}-e_{i}\right)-f(y)\right] \\
& +\sum_{i=1}^{d} \mu_{i}(y) y_{i}\left[f\left(y-e_{i}\right)-f(y)\right] .
\end{aligned}
$$

The next theorem states the main result on the ergodicity property of $Y$.

THEOREM 3.6. $Y$ is exponentially-ergodic and has a unique invariant probability measure $\pi_{x}$. Furthermore, function $S_{f}$ defined by

$$
\forall x \in \mathbb{R}^{+}, S_{f}(x)=\left\langle f, \pi_{x}\right\rangle,
$$

is locally Lipschitz continuous on $\mathbb{R}^{+}$, if, for some $p \in \mathcal{P}$ and $c>0,|f| \leq c\left(V_{p}+1\right)$.

We recall that

$$
\forall p \in \mathcal{P}, V_{p}(y)=\sum_{i=1}^{d}\left(\sum_{k=1}^{i} y_{k}\right)^{p_{i}} .
$$

To prove Theorem 3.6, we apply the criteria of [16]. Namely, we show below that $Y$ is irreducible (Lemma 3.7) by finding a path joining any pair of points in the state space, and we next show that function $V_{p}$ is a Lyapounov function for $Y$ (Lemma 3.8). Similarly to subsection 3.1, the main idea is to look at cumulative sums instead of individual numbers in each compartment.

LEMMA 3.7. For any $y, z \in \mathbb{N}^{d}$ :

$$
\mathbb{E}_{y}\left(\int_{0}^{\infty} \mathbb{1}_{Y_{t}=z} \mathrm{~d} t\right)>0
$$

Proof. Let $y, z \in \mathbb{N}^{d}$. We now construct a path $\mathcal{C}_{y}^{z}$ from $y$ to $z$ that involves $N$ events, such that the set

$$
A=\left\{\operatorname{choose} \mathcal{C}_{x}^{y} \text { among paths of } \mathrm{N} \text { events } \mid \text { at least } \mathrm{N} \text { events occur }\right\},
$$


has a positive probability. We further show that the time needed to perform at least $N$ events is finite almost surely, leading to the positive lower bound

$$
\mathbb{E}_{y}\left[\int_{0}^{\infty} 1_{Y_{t}=z} d t\right] \geq\left(\sum_{i=1}^{d}\left(\lambda_{i}(z)+\mu_{i}(z)\right)\right)^{-1} \mathbb{P}(A)>0
$$

since, when $Y_{t}$ reaches state $z$, it stays in $z$ for a mean time $\left(\sum_{i=1}^{d} \lambda_{i}(z)+\mu_{i}(z)\right)^{-1}>0$. To simplify the construction of $\mathcal{C}_{y}^{z}$, we use the same change of variables as in (3.2), with $v_{i}=\sum_{j=1}^{i} y_{j}$ and $w_{i}=\sum_{j=1}^{i} z_{j}$ (see Figure SM1):

(1) Total number compensation step. Starting from $v$, perform $M=\max \left(0, w_{1}-v_{1}, w_{2}-v_{2}, \ldots, w_{d}-v_{d}\right)$ times the immigration event of an individual into the first compartment, leading to state

$$
v^{(1)}=\left(v_{1}+M, v_{2}+M, \ldots, v_{d}+M\right) .
$$

These immigration events occur at rate $\lambda_{0}(\cdot) x>0$ (Hypothesis 2.2). Note that, by definition of $M, M \geq w_{k}-v_{k}$ for any $k$, and $v^{(1)} \geq w$ componentwise.

(2) Compartment-balancing step. For $k$ from 1 to $d-1$, starting from $v^{(k)}=$ $\left(w_{1}, \ldots, w_{k-1}, v_{k}+M, \ldots, v_{d}+M\right)$,

- If $M=w_{k}-v_{k}$, then $v^{(k)}=v^{(k+1)}$ and the state is left unchanged.

- If $M>w_{k}-v_{k}$, perform $M-\left(w_{k}-v_{k}\right)$ times the migration event from the $k^{t h}$ compartment into the next one to reach state $v^{(k+1)}$. For $i=M+v_{k}, \ldots, w_{k}+1$, each migration event occurs at rate $\lambda_{k}(\cdot)\left(i-w_{k-1}\right)$. Since $w_{k} \geq w_{k-1}$ and given Hypothesis 2.2, all above rates are strictly positive.

(3) Surplus-removing step. From state $v^{(d)}=\left(w_{1}, \ldots, w_{d-1}, v_{d}+M\right)$

- If $M=w_{d}-v_{d}$, then $v^{(d)}=w$ and the path has reached $z$.

- if $M>w_{d}-v_{d}$, perform $M-\left(w_{d}-v_{d}\right)$ times the death event in the last compartment to reach $w$. For $i=M+v_{d}, \ldots, w_{d}+1$, each event occurs at rate $\mu_{d}(\cdot)\left(i-w_{d-1}\right)$ and are strictly positive (Hypothesis 2.3).

The number $N$ of events in $\mathcal{C}_{y}^{z}$ is clearly finite, and can be bounded by $(d+1) M+\sum v_{k}$. Since we create no more than $M$ individuals along $\mathcal{C}_{y}^{z}$, the states stay in a compact of $\mathbb{N}^{d}$, and the total event rate is upper bounded. As the rate of each event occurring along $\mathcal{C}_{x}^{y}$ is strictly positive, we have $\mathbb{P}(A)>0$. Finally, as $Y$ has no absorption point (see Hypothesis 2.3), the time needed to perform at least $N$ events is clearly finite almost surely.

We now turn to the Lyapounov property.

LEMMA 3.8. $\forall p \in \mathcal{P}, V_{p}$ is a Lyapunov function for the generator $L_{x}$, that is: There exists a closed set $C \subset \mathbb{N}^{d}$ and $a_{1}, a_{2}>0$ such that:

$$
\forall y \in \mathbb{N}^{d}, L_{x} V_{p}(y) \leq-a_{1}\left[V_{p}(y)+1\right]+a_{2} \mathbb{1}_{C}(y) .
$$

Proof. Once again, we apply the change of variables (3.2) on the generator $L_{x}$ (with a slight abuse of notation): 


$$
\begin{aligned}
L_{x} V_{p}(w) & =\lambda_{0}(w) x\left[V_{p}\left(w+\sum_{i=1}^{d} e_{i}\right)-V_{p}(w)\right] \\
& +\sum_{i=1}^{d-1} \lambda_{i}(w)\left(w_{i}-w_{i-1}\right)\left[V_{p}\left(w-e_{i}\right)-V_{p}(w)\right] \\
& +\sum_{i=1}^{d} \mu_{i}(y)\left(w_{i}-w_{i-1}\right)\left[V_{p}\left(w-\sum_{j=i}^{d} e_{j}\right)-V_{p}(w)\right] .
\end{aligned}
$$

For any $i \in \llbracket 1, d \rrbracket, V_{p}\left(w-\sum_{j=i}^{d} e_{j}\right) \leq V_{p}\left(w-e_{i}\right) \leq V_{p}(w)$, and, given Hypotheses 2.1 and 2.3 , we have, for any $w \in \mathcal{S}$ :

$$
L_{x} V_{p}(w) \leq B_{0} x \sum_{i=0}^{d}\left[\left(w_{i}+1\right)^{p_{i}}-w_{i}^{p_{i}}\right]+\sum_{i=1}^{d} \alpha_{i}\left(w_{i}-w_{i-1}\right)\left[\left(w_{i}-1\right)^{p_{i}}-w_{i}^{p_{i}}\right]
$$

The same algebraic manipulations as in (3.8) lead to Lemma 3.8.

Proof of Theorem 3.6. Combining Lemmas 3.7 and 3.8, with [16, Theorem 4.4], we obtain that $Y$ has a unique invariant probability measure $\pi_{x}$, and, moreover, for any $p \in \mathcal{P}$,

$$
\left\langle V_{p}, \pi_{x}\right\rangle<\infty
$$

To show that $S_{f}$ is locally Lipschitz continuous, we first prove that function $S_{V_{p}}$ is locally bounded, for any $p \in \mathcal{P}$, by a coupling approach. Let $V$ be the cumulative sums associated with $Y$ (see (3.2)), and let $x \in(0, K)$ with $K<\infty$. By analogy with (3.4), let $W$ be the càdlàg d-dimensional Markov process defined in law by the following generator:

$$
B g(w)=B_{0} K\left[g\left(w+\sum_{i=1}^{d} e_{i}\right)-g(w)\right]+\sum_{j=1}^{d} \alpha_{i}\left(w_{i}-w_{i-1}\right)\left[g\left(w-e_{i}\right)-g(w)\right] .
$$

We take $W(0) \geq V(0)$. Since $\lambda_{0}(\cdot) x<B_{0} K$, adapting the coupling used to prove Lemma 3.3, we have that, for any $t>0$, for any $i \in \llbracket 1, d \rrbracket, V_{i}(t) \leq W_{i}(t)$ stochastically. Moreover $W$ is also clearly exponentially ergodic, and thanks to Lyapounov function $V_{p}$, its stationary measure $\nu_{K}$ satisfies, for any $x<K$, any $p \in \mathcal{P}$,

$$
\left\langle V_{p}, \pi_{x}\right\rangle \leq\left\langle V_{p}, \nu_{K}\right\rangle<\infty \text {. }
$$

Let $f$ be a function such that $|f| \leq c\left(V_{p}+1\right)$ for some $p \in \mathcal{P}$ and $c>0$. It remains to show that function $S_{f}$ is locally Lipschitz continuous on $\mathbb{R}^{+}$. We introduce the Poisson equation associated with $\left(f, L_{x}\right)$ :

$$
L_{x} h=\left\langle f, \pi_{x}\right\rangle-f,
$$

and, for any function $h: \mathbb{N}^{d} \mapsto \mathbb{R}$, the $\left(V_{p}+1\right)$-norm

$$
\|h\|_{V+1}:=\sup _{x \in \mathbb{N}^{d}} \frac{|h(x)|}{V_{p}(x)+1} .
$$


Thanks to the Lyapounov property Lemma 3.8, we can apply [11, Theorem 3.2] to obtain that, for any $x>0$, there exists $\alpha_{x}<\infty$ such that (3.11) admits a solution, $g_{x}$, with $\left|g_{x}\right| \leq \alpha_{x}\left(V_{p}+1\right)$. From (3.10), for any $x_{1}, x_{2} \in \mathbb{R}^{+}, \pi_{x_{2}}\left(g_{x_{1}}\right)<\infty$, and we have:

$$
\begin{aligned}
\left\langle f, \pi_{x_{2}}-\pi_{x_{1}}\right\rangle & =\left\langle f, \pi_{x_{2}}\right\rangle-\left\langle f, \pi_{x_{1}}\right\rangle\left\langle\mathbb{1}, \pi_{x_{2}}\right\rangle=\left\langle f-\left\langle f, \pi_{x_{1}}\right\rangle \mathbb{1}, \pi_{x_{2}}\right\rangle \\
& =\left\langle-L_{x_{1}} g_{x_{1}}, \pi_{x_{2}}\right\rangle=\left\langle-L_{x_{1}} g_{x_{1}}+L_{x_{2}} g_{x_{1}}, \pi_{x_{2}}\right\rangle .
\end{aligned}
$$

From (2.2), for any $y \in \mathbb{N}^{d}$,

$$
-L_{x_{1}} g_{x_{1}}(y)+L_{x_{2}} g_{x_{1}}(y)=\lambda_{0}(y)\left(x_{2}-x_{1}\right)\left[g_{x_{1}}\left(y+e_{1}\right)-g_{x_{1}}(y)\right] .
$$

Since $g_{x_{1}}$ has a finite $(V+1)$-norm, it is also true for $\tilde{g}_{x_{1}}: y \mapsto g_{x_{1}}\left(y+e_{1}\right)$, hence $\pi_{x_{2}}\left(\tilde{g}_{x_{1}}\right)<\infty$, and we have

$$
\left\langle f, \pi_{x_{2}}-\pi_{x_{1}}\right\rangle=\left(x_{2}-x_{1}\right)\left\langle\left(\tilde{g}_{x_{1}}-g_{x_{1}}\right) \lambda_{0}, \pi_{x_{2}}\right\rangle .
$$

Let $\mathrm{K}$ be a neighborhood of $x_{1}$, we obtain that, for any $x_{2} \in K$,

$$
\left|\left\langle f, \pi_{x_{2}}-\pi_{x_{1}}\right\rangle\right| \leq\left|x_{2}-x_{1}\right| \max \left(\left\|\tilde{g}_{x_{1}}\right\|_{V_{p}+1},\left\|g_{x_{1}}\right\|_{V_{p}+1}\right) \times 2 B_{0} \sup _{y \in K}\left\langle V_{p}, \pi_{y}\right\rangle,
$$

which concludes the proof.

4. Proof of the main result. In this section, we will prove Theorem 2.6 in three steps: relative compactness of $\left(X^{\varepsilon}, Y^{\varepsilon}\right)$ in an appropriate topology, identification of a limiting equation for any adherence value, and uniqueness of the limit. The first two steps rely on the tools developed in [14] with the estimates provided by Proposition 3.1. The last step relies on Theorem 3.6.

4.1. Relative compactness. We start by the relative compactness of $X^{\varepsilon}$ in $\mathcal{D}_{\mathbb{R}^{+}}\left[0, \infty\left[\right.\right.$. Let $A: \mathcal{C}_{b}^{2}\left(\mathbb{R}^{+}\right) \rightarrow \mathcal{C}_{b}^{1}\left(\mathbb{R}^{+} \times \mathbb{N}^{d}\right)$ be the infinitesimal generator defined for any $x \in \mathbb{R}^{+}$and $y \in \mathbb{N}^{d}$ by:

$$
A f(x, y)=\left(r_{0}(y)-\lambda_{0}(y)-\mu_{0}(y)\right) x f^{\prime}(x)
$$

Then, using Definition 2.5 with $g$ depending only on the first variable, i.e. $\forall y \in$ $\mathbb{N}^{d}, g(\cdot, y)=f(\cdot)$, we obtain that

$$
M_{f}^{\varepsilon}(t)=f\left(X^{\varepsilon}(t)\right)-f\left(X^{\varepsilon}(0)\right)-\int_{0}^{t} A f\left(X^{\varepsilon}(s), Y^{\varepsilon}(s)\right) \mathrm{d} s+R_{f}^{\varepsilon}(t)
$$

is a $\mathcal{F}_{t}^{\varepsilon}$-martingale, where $R_{f}^{\varepsilon}(t)=\int_{0}^{t}\left[A f-A^{\varepsilon} f\right]\left(X^{\varepsilon}(s), Y^{\varepsilon}(s)\right) \mathrm{d} s$. Applying [6, Theorems 3.9 .1 and 3.9.4], $X^{\varepsilon}$ is relatively compact in $\mathcal{D}_{\mathbb{R}^{+}}[0, \infty[$ if, for any $f \in \mathcal{C}_{b}^{2}\left(\mathbb{R}^{+}\right)$:

- $\mathbb{E}\left(\sup _{t \leq T}\left|R_{f}^{\varepsilon}(t)\right|\right) \underset{\varepsilon \rightarrow 0}{\longrightarrow} 0$,

- $\exists q>1$ such that $\sup _{\varepsilon} \mathbb{E}\left[\int_{0}^{T}\left|A f\left(X^{\varepsilon}(s), Y^{\varepsilon}(s)\right)\right|^{q} \mathrm{~d} s\right]<\infty$. 
With $f \in \mathcal{C}_{b}^{2}$, we can apply the Taylor-Lagrange formula:

$$
\sup _{t \leq T}\left|R_{f}^{\varepsilon}(t)\right| \leq \frac{\varepsilon}{2}\left\|f^{\prime \prime}\right\|_{\infty} \int_{0}^{T} X^{\varepsilon}(s)\left(R_{0}+B_{0}+D_{0}\left[1+\left(Y_{\text {tot }}^{\varepsilon}(s)\right)^{m_{0}}\right]\right) \mathrm{d} s,
$$

given Hypotheses 2.1, 2.2, and 2.4. Let $T^{\varepsilon}(t)=X^{\varepsilon}(t)+\sum_{i=1}^{d} Y_{i}^{\varepsilon}(t)$. From Proposition 3.1, there exists $C_{1}>0$ independent of $\varepsilon$ such that:

$$
\mathbb{E}\left(\sup _{t \leq T}\left|R_{f}^{\varepsilon}(t)\right|\right) \leq \frac{\varepsilon T}{2}\left\|f^{\prime \prime}\right\|_{\infty} C_{1}\left(1+\sup _{\varepsilon} \sup _{s \leq T} \mathbb{E}\left[\left|T^{\varepsilon}(s)\right|^{m_{0}+1}\right]\right) \underset{\varepsilon \rightarrow 0}{\longrightarrow} 0 .
$$

Also, from Proposition 3.1, for any $q>1$, there exists, with similar calculations, $C_{2}>0$ independent of $\varepsilon$ such that:

$$
\mathbb{E}\left[\int_{0}^{T}\left|A f\left(X^{\varepsilon}(s), Y^{\varepsilon}(s)\right)\right|^{q} \mathrm{~d} s\right] \leq\left\|f^{\prime}\right\|_{\infty}^{q} C_{2} T\left[1+\sup _{\varepsilon} \sup _{s \leq T} \mathbb{E}\left(\left|T^{\varepsilon}(s)\right|^{q\left(m_{0}+1\right)}\right)\right] \underset{\varepsilon \rightarrow 0}{\longrightarrow} 0 .
$$

We turn to the compactness of $Y^{\varepsilon}$, which does not occur in a functional sense. Let $\Gamma_{\varepsilon}$ be the occupation measure of $Y^{\varepsilon}$ over time, defined by:

$$
\forall A \subset \mathbb{N}^{d}, \Gamma_{\varepsilon}([0, t] \times A)=\int_{0}^{t} \mathbb{1}_{A}\left(Y^{\varepsilon}(s)\right) \mathrm{d} s
$$

It is clear that $\Gamma_{\varepsilon} \in \mathcal{L}_{m}\left(\mathbb{N}^{d}\right)$, the space of measure $\mu$ on $\left[0, \infty\left[\times \mathbb{N}^{d}\right.\right.$ such that $\mu\left(\left[0, t\left[\times \mathbb{N}^{d}\right)=t\right.\right.$. For any $K>0$, from Proposition 3.1 and the Markov inequality,

$$
\mathbb{P}\left(Y_{\text {tot }}^{\varepsilon}(t) \geq K\right) \leq \frac{\sup _{\varepsilon} \sup _{t>0} \mathbb{E}\left(Y_{\text {tot }}^{\varepsilon}(t)\right)}{K}
$$

For any $\nu>0$, there exists a $K>0$, such that, for all $t>0$ and $\varepsilon>0$,

$$
\mathbb{P}\left(Y_{\text {tot }}^{\varepsilon}(t) \leq K\right) \geq 1-\nu
$$

hence with $B_{K}=\left\{y \in \mathbb{N}^{d}, y_{t o t} \leq K\right\}, \inf _{\varepsilon} \mathbb{E}\left(\Gamma_{\varepsilon}\left([0, t] \times B_{K}\right)\right) \geq t(1-\nu)$, and we can conclude with $[14$, Lemma 1.3$]$ that $\left(X^{\varepsilon}, \Gamma_{\varepsilon}\right)$ is relatively compact in $\mathcal{D}_{\mathbb{R}^{+}}\left[0, \infty\left[\times \mathcal{L}_{m}\left(\mathbb{N}^{d}\right)\right.\right.$.

4.2. Identification of the limit. Let $(x, \Gamma)$ be a limiting point (up to a subsequence) of $\left(X^{\varepsilon}, \Gamma_{\varepsilon}\right)$ in $\mathcal{D}_{\mathbb{R}^{+}}\left[0, \infty\left[\times \mathcal{L}_{m}\left(\mathbb{N}^{d}\right)\right.\right.$. As the first component $X^{\varepsilon}$ has vanishing jump sizes, namely (a.s.) for all $T>0$,

$$
\sup _{t \in[0, T]}\left|X^{\varepsilon}(t)-X^{\varepsilon}\left(t^{-}\right)\right| \leq \varepsilon \rightarrow 0
$$

$x$ is clearly a continuous function.

We start by identifying the limit measure $\Gamma$. Using Definition 2.5 with $g$ depending 
only on the second variable, i.e. $\forall x \in \mathbb{R}^{+}, g(x, \cdot)=h(\cdot)$, we have

$$
\begin{aligned}
A^{\varepsilon} h(x, y)= & \frac{\lambda_{0}(y)}{\varepsilon} x\left[h\left(y+e_{1}\right)-h(y)\right]+\sum_{i=1}^{d-1} \frac{\lambda_{i}(y)}{\varepsilon} y_{i}\left[h\left(y+e_{i+1}-e_{i}\right)-h(y)\right] \\
& +\sum_{i=1}^{d} \frac{\mu_{i}(y)}{\varepsilon} y_{i}\left[h\left(y-e_{i}\right)-h(y)\right] \\
= & \frac{1}{\varepsilon} L_{x} h(y),
\end{aligned}
$$

according to (2.2). By definition of the infinitesimal generator, the process

$$
\varepsilon\left[h\left(Y^{\varepsilon}(t)\right)-h\left(Y^{\varepsilon}(0)\right)\right]-\int_{0}^{t} \int_{\mathbb{N}^{d}} L_{X^{\varepsilon}(s)} h(y) \Gamma_{\varepsilon}(\mathrm{d} s, \mathrm{~d} y),
$$

is a $\mathcal{F}_{t}^{\varepsilon}$-martingale. Applying [14, Lemma $1.5 \mathrm{~d}$ ], since rates are polynomial (Hypothesis 2.4) and using Proposition 3.1, we have, for any $h \in \mathcal{C}_{b}^{2}\left(\mathbb{N}^{d}\right)$,

$$
\lim _{\varepsilon \rightarrow 0} \int_{0}^{t} \int_{\mathbb{N}^{d}} L_{X^{\varepsilon}(s)} h(y) \Gamma_{\varepsilon}(\mathrm{d} s, \mathrm{~d} y)=\int_{0}^{t} \int_{\mathbb{N}^{d}} L_{x(s)} h(y) \Gamma(\mathrm{d} s, \mathrm{~d} y) .
$$

From the martingale (4.2), it follows that

$$
\int_{0}^{t} \int_{\mathbb{N}^{d}} L_{x(s)} h(y) \Gamma(\mathrm{d} s, \mathrm{~d} y)
$$

is a continuous martingale of bounded variations, hence it is identically zero, for any $h \in \mathcal{C}_{b}^{2}\left(\mathbb{N}^{d}\right)$ and with probability one. Let $\nu$ be the measure defined by $\nu([0, t])=$ $\Gamma\left([0, t], \mathbb{N}^{d}\right)$ for all $t$. From [14, Lemma 1.4], there exists a process $\gamma$ such that, for any $h \in \mathcal{C}_{b}^{2}\left(\mathbb{N}^{d}\right)$,

$$
\int_{0}^{t} \int_{\mathbb{N}^{d}} L_{x(s)} h(y) \Gamma(\mathrm{d} s, \mathrm{~d} y)=\int_{0}^{t} \int_{\mathbb{N}^{d}} L_{x(s)} h(y) \gamma_{s}(\mathrm{~d} y) \nu(\mathrm{d} s) .
$$

With the same arguments as in [14, Example 2.3], we have, almost surely,

$$
\int_{\mathbb{N}^{d}} L_{x(t)} h(y) \gamma_{t}(\mathrm{~d} y)=0,
$$

for all $t$ outside of a set of zero Lebesgue measure. Since $Y$ has a unique invariant probability measure (Theorem 3.6), we get $\gamma_{t}=\pi_{x(t)}$, and $\Gamma(\mathrm{d} s, \mathrm{~d} y)=\pi_{x(\mathrm{~d} s)}(\mathrm{d} y) \mathrm{d} s$.

It only remains to identify function $x$. With the martingale $M_{f}^{\varepsilon}(t)$ defined in (4.1), and with $f=I d$, taking the limit $\varepsilon \rightarrow 0$, there exists a filtration $\left(\mathcal{F}_{t}\right)_{t}$ such that (applying [14, Lemma $1.5 \mathrm{~d}]$ again):

$$
M_{t}=x(t)-x^{i n}-\int_{0}^{t} \int_{\mathbb{N}^{d}} A I d(x(s), y) \pi_{x(\mathrm{~d} s)}(\mathrm{d} y) \mathrm{d} s,
$$

is a $\left(\mathcal{F}_{t}\right)_{t}$-martingale. For $s>0$,

$$
\begin{aligned}
\int_{\mathbb{N}^{d}} A I d(x(s), y) \pi_{x(s)}(\mathrm{d} y) & =x(s) \int_{\mathbb{N}^{d}}\left[r_{0}(y)-\lambda_{0}(y)-\mu_{0}(y)\right] \pi_{x(s)}(\mathrm{d} y) \\
& =x(s) \Lambda_{0}(x(s)) .
\end{aligned}
$$


Furthermore, according to (4.1), $M_{I d}^{\varepsilon}$ is a $\mathcal{F}_{t}^{\varepsilon}$-martingale with predictable quadratic variation

$$
\left\langle M_{I d}^{\varepsilon}\right\rangle_{t}=\varepsilon \int_{0}^{t}\left[r_{0}\left(Y^{\varepsilon}(s)\right)+\lambda_{0}\left(Y^{\varepsilon}(s)\right)+\mu_{0}\left(Y^{\varepsilon}(s)\right)\right] \mathrm{d} s .
$$

Using Proposition 3.1, $\mathbb{E}\left(\left\langle M_{I d}^{\varepsilon}\right\rangle_{t}\right) \underset{\varepsilon \rightarrow 0}{\longrightarrow} 0$. Hence, $M_{t} \equiv 0$ for all times $t$, and $x$ is solution of

$$
x(t)=x^{i n}+\int_{0}^{t} \Lambda_{0}(x(s)) x(s) \mathrm{d} s .
$$

Since $S_{f}$ is continuous for any $|f| \leq c\left(V_{p}+1\right)$ (see Theorem 3.6), we deduce by composition that $x \in \mathcal{C}^{1}\left(\mathbb{R}^{+}\right)$, and is solution of:

$$
\frac{\mathrm{d} x}{\mathrm{~d} t}(t)=\Lambda_{0}(x(t)) x(t), \quad x(0)=x^{i n} .
$$

4.3. Uniqueness of the limit. With Theorem 3.6, we have proved that, for any limiting point $x$ of $X^{\varepsilon}$, the limit of the fast process $Y^{\varepsilon}$ is uniquely determined by the invariant probability measure $\pi_{x}$ of $L_{x}$. It remains to prove the uniqueness of $x$, as a solution of

$$
\frac{\mathrm{d} z}{\mathrm{~d} t}=\Lambda_{0}(z) z, \quad z(0)=x^{i n} .
$$

We will prove that the right-hand side function $F: \mathbb{R}^{+} \rightarrow \mathbb{R}$ defined by

$$
F(x)=x\left\langle r_{0}-\lambda_{0}-\mu_{0}, \pi_{x}\right\rangle,
$$

is locally Lipschitz continuous. Let $x_{1} \in \mathbb{R}^{+}$and $U \subset \mathbb{R}^{+}$a neighborhood of $x_{1}$. From Hypotheses 2.1 and 2.4, we get, for any $x_{2} \in U$ :

$$
\begin{aligned}
\left|F\left(x_{1}\right)-F\left(x_{2}\right)\right| & =\left|\left\langle r_{0}-\lambda_{0}-\mu_{0}, \pi_{x_{1}}-\pi_{x_{2}}\right\rangle x_{1}+\left\langle r_{0}-\lambda_{0}-\mu_{0}, \pi_{x_{2}}\right\rangle\left(x_{1}-x_{2}\right)\right| \\
& \leq x_{1}\left|\left\langle r_{0}-\lambda_{0}-\mu_{0}, \pi_{x_{2}}-\pi_{x_{1}}\right\rangle\right| \\
& +\left|x_{1}-x_{2}\right|\left(R_{0}+B_{0}+D_{0}\right)\left\langle 1+\left(y_{t o t}\right)^{m_{0}}, \pi_{x_{2}}\right\rangle .
\end{aligned}
$$

Choosing $p=\left(m_{0}+d-1, m_{0}+d-2, \ldots, m_{0}\right) \in \mathcal{P}, 1+\left(y_{\text {tot }}\right)^{m_{0}} \leq 1+V_{p}$. From Hypothesis 2.4 and Theorem 3.6, there exists $C_{1}>0$ (depending on $x_{1}$ ) such that

$$
\forall x_{2} \in U,\left|F\left(x_{1}\right)-F\left(x_{2}\right)\right| \leq C_{1}\left|x_{1}-x_{2}\right|
$$

$F$ is thus locally Lipschitz continuous on $\mathbb{R}^{+}$, and we can deduce the uniqueness of a maximal solution of (4.3) on $\mathbb{R}^{+}$from the Cauchy-Lipschitz theorem. Since $\Lambda_{0}$ is upper-bounded by $R_{0}$, we deduce from the comparison principle that $x$ is a global solution of (4.3).

5. Numerical illustration and biological application . In this section, we present some results on the linear version of the model (with constant rates), and on the identifiability of the model parameters. We then detail an algorithm suited for simulating $\pi_{x}$, which we use to illustrate the numerical convergence. Finally, we compare the model behavior in different parameter regimes specifically designed for our application to ovarian follicle development. 
5.1. Linear case. Let us consider the process $\left(X^{\varepsilon}(t), Y^{\varepsilon}(t)\right)_{t \geq 0}$ (see Definition 2.5) with all event rates $\lambda_{i}, \mu_{i}$ fixed to constant values. From Theorem 2.6, we have:

Proposition 5.1 (Linear case). For any initial condition $\left(X^{\varepsilon}(0), Y^{\varepsilon}(0)\right) \in$ $\left(\varepsilon \mathbb{N} \times \mathbb{N}^{d}\right)$ such that, for some $x^{\text {in }} \in \mathbb{R}^{+}$,

$$
\forall k>1, \sup _{\varepsilon} \mathbb{E}\left(X^{\varepsilon}(0)^{k}\right)<\infty, \sup _{\varepsilon} \mathbb{E}\left(Y_{\text {tot }}^{\varepsilon}(0)^{k}\right)<\infty, X^{\varepsilon}(0) \underset{a . s}{\longrightarrow} x^{i n}
$$

the process $\left(X^{\varepsilon}, Y^{\varepsilon}\right)$ has a unique limit process $(x, \pi) \in \mathcal{C}^{1}\left(\mathbb{R}^{+}\right) \times \mathcal{L}_{m}\left(\mathbb{N}^{d}\right)$ :

$$
\left\{\begin{array}{ll}
x(t) & =x^{i n} \exp \left(\left(r_{0}-\lambda_{0}-\mu_{0}\right) t\right) \\
\pi_{x(t)}(y) & =\prod_{i=1}^{d} \frac{\left(x(t) \alpha_{i}\right)^{y_{i}}}{y_{i} !} e^{-x(t) \alpha_{i}}
\end{array},\right.
$$

with $\alpha_{i}=\prod_{j=0}^{i-1} \frac{\lambda_{j}}{\lambda_{j+1}+\mu_{j+1}}$ for $1 \leq i \leq d$.

Proof. The result follows directly from Theorem 2.6, and from the forward Kolmogorov equation applied to the fast process $Y$ [18, Thm 2.4].

Suppose now we have $\bar{x}(t)$ and $\left(\bar{y}_{j}(t)\right)_{1<j<d}$ the number of individuals (e.g. follicles) observed in each compartment at any time between 0 and $T_{f}$. Since the number $x$ of individuals in the slow compartment (reserve) has an analytic expression, we can identify $\Lambda_{0}$ and $x^{i n}$ from $(\bar{x}(t))_{0 \leq t \leq T_{f}}$ for large $T_{f}$. Following [24], we use the following quantities to compute $x^{i n}$ and $\Lambda_{0}$ :

$$
\begin{aligned}
\int_{0}^{T_{f}} \bar{x}(s) \mathrm{d} s & =\frac{x^{i n}}{\Lambda_{0}}\left(1-\exp \left(-\Lambda_{0} T_{f}\right)\right) \sim \frac{x^{i n}}{\Lambda_{0}} \\
\int_{0}^{T_{f}} s \bar{x}(s) \mathrm{d} s & =\frac{x^{i n}}{\Lambda_{0}{ }^{2}}-\frac{x^{i n}}{\Lambda_{0}}\left(T_{f}+\frac{1}{\Lambda_{0}}\right) \exp \left(-\Lambda_{0} T_{f}\right) \sim \frac{x^{i n}}{\Lambda_{0}{ }^{2}} .
\end{aligned}
$$

To identify separately the exhaustion rate $\lambda_{0}+\mu_{0}$ of the first compartment from the renewing rate $r_{0}$, we need additional observations to distinguish between outgoing and from births.

To estimate the remaining parameters, $\left(\alpha_{i}\right)_{1 \leq i \leq d}$, we apply the maximum likelihood principle to the Poisson distribution of each compartment $j$, for any $1 \leq j \leq d$ $\left(\left(\bar{y}_{j}(t)\right)_{0 \leq t \leq T_{f}}\right)$, and obtain an estimate $\bar{\alpha}_{j}$ of $\alpha_{j}$ as:

$$
\bar{\alpha}_{j}=\left(\int_{0}^{T_{f}} \bar{y}_{j}(t) \mathrm{d} t\right) /\left(\int_{0}^{T_{f}} \bar{x}(t) \mathrm{d} t\right) .
$$

To identify separately the $\lambda_{i}$ s from the $\mu_{i} \mathrm{~s}$, additional observations would be needed on the number of deaths occurring in each compartment, or the transit times from one compartment to the next.

5.2. Strategy to compute $\pi_{x}$ and the limit model. We use an Euler scheme over the time range $[0,5]$ (with a time step of $5 \times 10^{-3}$ ) to simulate the ordinary differential equation ruling the dynamics of the first compartment. To evaluate the right hand side, the scheme requires the value of $\Lambda_{0}$ at each time step, given the current value of $x$ (Figure 1).

Let $x \in \mathbb{R}^{+}, N_{0} \in \mathbb{N}^{*}$ and $T_{f}>0$. Algorithm 5.1 computes both the expectation $E$ 


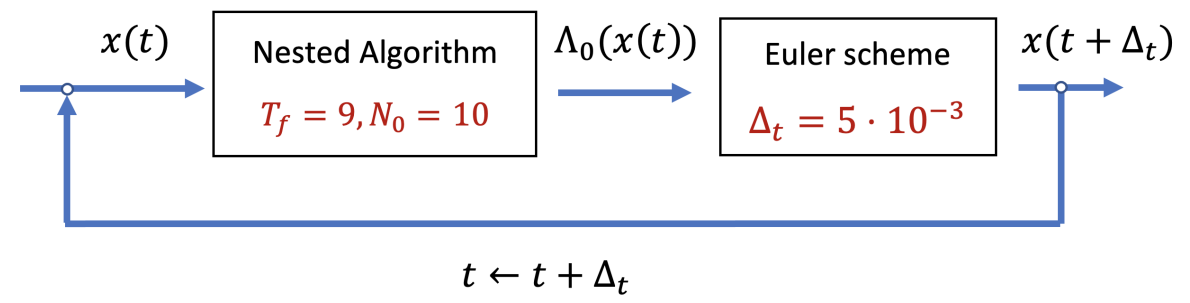

FIG. 1. Main steps of the limit model simulation

of $\pi_{x}$, and the corresponding rate $\Lambda_{0}(x)=\sum_{y \in \mathbb{N}^{d}}\left(r_{0}(y)-\lambda_{0}(y)-\mu_{0}(y)\right) \pi_{x}(y)$. The Markov chain associated with the fast process is first simulated $N_{0}$ times over the time range $\left[0, T_{f}\right]$ by running an exact simulation algorithm [10], then the computed trajectories (time and location $\left(t_{i}\right)_{i},\left(Y_{t_{i}}\right)_{i}$ at each jump in $\left[0, T_{f}\right]$ ) are used to estimate $\pi_{x}$. The values of $T_{f}$ and $N_{0}$ are a good compromise between accuracy (owing to [23], the error on $\lambda_{0}$ can be arbitrary small for large $T_{f}$ and $N_{0}$ ) and speed.

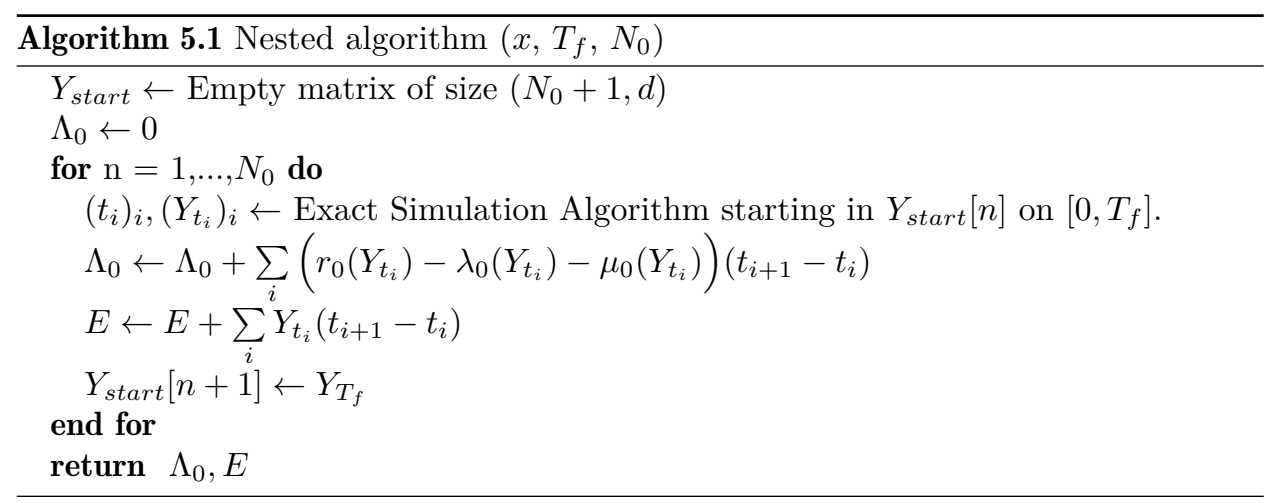

5.3. Parameter values. We specify the formulation of the event rates as proposed in [1] for a toy model of mammal follicle development. The numerical study performed here does not intend to reproduce accurately the follicle numbers in a specific species, and rather gives semi-quantitative insight into the follicle population dynamics. We focus on the control of the exit from the reserve (given $r_{0}=0$ ) by the growing follicles $(d=3)$, with $\lambda_{0}(y)$ expressed as:

$$
\lambda_{0}(y)=a+\frac{b}{1+c\left(y_{1}+y_{2}+y_{3}\right)}
$$

A schematic scheme of the $3 d$ process $(X, Y) \in \mathcal{D}_{\mathbb{R}^{+} \times \mathbb{N}^{3}}[0, \infty[$ is provided in Figure SM2. We instantiated the parameter values in $\lambda_{0}(y)$ so that the control exerted onto the reserve be operating (see Figure SM3): $a=0.1, b=1.5, c=0.01$. All other rates are fixed to constant values $\lambda_{1}(y)=\lambda_{2}(y)=0.5, \mu_{0}(y)=\mu_{1}(y)=\mu_{2}(y)=0.3$, $\mu_{3}(y)=0.2$, in such a way that the distribution $\pi_{x}$ of the limit model is nonmonotonous (see Figure SM4), as observed for growing follicles [21]. At initial time, the growing compartments are empty, and the reserve size is set to $x^{i n}=10^{2}$.

5.4. Illustration of the convergence. Figure 2 illustrates the convergence of the sequence $\left(X^{\varepsilon}, Y_{1}^{\varepsilon}, Y_{2}^{\varepsilon}, Y_{3}^{\varepsilon}\right)$ to $(x, \pi)$ as $\varepsilon \rightarrow 0$ (Theorem 2.6). The trajectories of the 
initial, full model (Definition 2.5) are obtained by averaging $10^{4}$ runs of an exact stochastic algorithm for different values of $\varepsilon$, and compared to the limit model. To improve the estimate of the mean of $\pi_{x}$, we average the output of the Euler scheme combined with Algorithm 5.1 on $10^{3}$ runs. We quantify the convergence rate (top right panel) using the $l^{1}$ norm error at time $t, E_{1}$, and the total $l^{1}$ norm, $E_{T}$ :

$$
E_{1}(t)=\left|\mathbb{E} X_{0}^{\varepsilon}(t)-x(t)\right|+\sum_{i=1}^{3}\left|\mathbb{E} Y_{i}^{\varepsilon}(t)-\mathbb{E}\left\langle x \mapsto x_{i}, \pi_{t}\right\rangle\right|, \quad E_{T}=\int_{0}^{T} E_{1}(t) \mathrm{d} t
$$
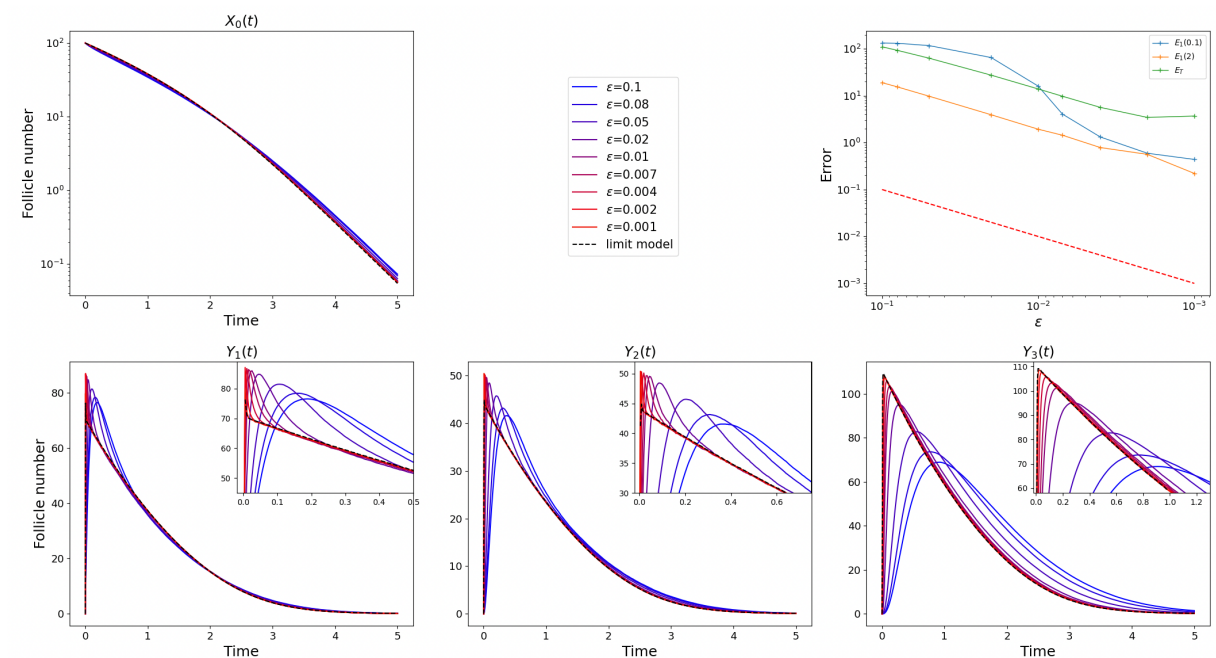

FIG. 2. Convergence to the limit model

Changes in the number $X^{\varepsilon}$ of follicles in the reserve (top left panel) and in the number $\left(Y_{i}^{\varepsilon}\right)_{1 \leq i \leq 3}$ of growing follicles (bottom panels) for different values of $\varepsilon$ (see legend insert). Top right panel: $l^{1}$ norm error $E_{1}(t)$ at time $t=0.1$ (blue line) or $t=2$ (orange line), and total $l^{1}$ norm $E_{T}$ (green line) as a function of $\varepsilon$. The red dotted line provides a reference to an order 1 error in $\varepsilon$.

The total $l^{1}$ norm error decreases linearly with $\varepsilon$. This is also the case of the $l^{1}$ norm error after the transient regime (as for $E_{1}(2)$ at time $t=2$ ), while the $\varepsilon$ dependent pattern at earlier times (typically from time 0 to 0.5 , as for $E_{1}(0.1)$ at time $t=0.1)$ ) is still decreasing, yet nonlinearly.

5.5. Biological model outputs . The status of the ovaries is mainly characterized by the number of quiescent follicles, as a function of age, on one side, and the distribution of growing follicles into different maturation stages, on the other side.

We now illustrate how the model outputs can participate in investigating two main issues : (i) What is the kinetics of the reserve decline up to (quasi-)exhaustion?, and (ii) What does the growing follicle distribution look like and is its shape preserved with aging?

We tackle the first question by studying the impact of the nonlinear formulation on the decay rate of the reserve, in comparison with linear formulations. To compare the linear and nonlinear formulations, we focus not only on the decrease in the number $x$ of follicles in the reserve, but also on the cumulative number $E_{3}^{T}=\int_{0}^{T} \mu_{3} Y_{3}(s) \mathrm{d} s$ of follicles exiting from the last compartment. $E_{3}^{T}$ is a marker of performance of the reproduction process and can be assimilated to the number of ovulations occurring all along reproductive lifespan. To perform the comparison, we first simulate the 
nonlinear model with the same set of parameters as in subsection 5.2 to obtain synthetic data. We then fit the linear model to these data in three different ways (the parameter values are gathered in Table 2). In the first two cases, we first estimate the meta-parameters $\left(\bar{\alpha}_{i}\right)_{1 \leq i \leq 3}(5.2)$, and then the parameters affecting the reserve dynamics, $x^{i n}$ and the decay rate $\lambda_{0}+\mu_{0}$, using either a linear regression on $\ln (x)$, or the analytic expression of $x(t)$ (5.1). In the third case, we keep all parameters identical to the nonlinear formulation, except $\lambda_{0}$, whose value is retrieved from the least-squares fitting of the $\left(\bar{\alpha}_{i}\right)_{1 \leq i \leq 3}$. Finally, we compute the expectation, variance and coefficient of variation of the normalized numbers of growing follicles $\left(\left(Y_{i} / x\right)_{i=1,2,3}\right)$, numerically in the nonlinear case or analytically, using the intensities of the Poisson laws in Proposition 5.1 in the linear case).

TABLE 2

Parameter values used to compare the linear and non linear formulations of the model. Estimated values are in red. For the nonlinear model, the meta-parameters $\left(\bar{\alpha}_{i}\right)_{1<i<3}$ are estimated using (5.2). These values are left unchanged for versions 1 and 2. For version 3, the value of $\lambda_{0}$ is retrieved from the least-squares fitting of the $\left(\bar{\alpha}_{i}\right)_{1 \leq i \leq 3}$.

\begin{tabular}{|c|c|c|c|c|}
\hline & \multirow{2}{*}{ Nonlinear model } & \multicolumn{3}{|c|}{ Linear models } \\
\hline & & version 1 & version 2 & version 3 \\
\hline $\begin{array}{l}\text { Estimation } \\
\text { method }\end{array}$ & / & $\begin{array}{l}\text { linear regression } \\
\text { on } \ln (x)\end{array}$ & Integrals (5.1) & $\begin{array}{l}\text { Estimation of } \lambda_{0} \\
\text { from }\left(\bar{\alpha}_{i}\right)_{1 \leq i \leq 3}\end{array}$ \\
\hline \multirow{3}{*}{$\left(\alpha_{i}\right)_{1 \leq i \leq 3}$} & $\bar{\alpha}_{1}=0.9490$ & $\bar{\alpha}_{1}$ & $\bar{\alpha}_{1}$ & $\alpha_{1}=0.9487$ \\
\hline & $\bar{\alpha}_{2}=0.5931$ & $\bar{\alpha}_{2}$ & $\bar{\alpha}_{2}$ & $\alpha_{2}=0.5930$ \\
\hline & $\bar{\alpha}_{3}=1.4821$ & $\bar{\alpha}_{3}$ & $\bar{\alpha}_{3}$ & $\alpha_{3}=1.4824$ \\
\hline$x^{i n}$ & 100 & 177.7 & 116.7 & 100.0 \\
\hline$\lambda_{0}+\mu_{0}$ & $0.1+\frac{1.5}{1+0.01\left(y_{1}+y_{2}+y_{3}\right)}+0.3$ & 1.532 & 1.237 & $0.758+0.3$ \\
\hline
\end{tabular}

The fitting results are illustrated on Figure 3. No linear version is able to capture the whole dynamics. Version 1 fits the reserve size well, yet there is a precocious shift on the cumulative number of follicles exiting from $Y_{3}$. In contrast, this output is rather well fitted by versions 1 and 2 , yet at the expenses of a poorer fit to $x(t)$.

The effect of the nonlinear formulation is clearly seen on the pattern of the reserve dynamics. Indeed, the decay rate is not constant. It increases around the mid-simulation time, as a result of the decreasing number of follicles in the growing compartments, which progressively relaxes the feedback exerted onto the activation rate. Looking at the functional response given by the function $x \mapsto \Lambda_{0}(x)$, one has a direct access to the impact of growing follicles onto the reserve kinetics. In particular, the model results give mechanistic insight into statistical approaches that use nonlinear regression models to fit experimental data on the reserve size according to age in mammals (reviewed in [3], see also Figure SM3).

The impact of nonlinear rates is further illustrated on Figure 4. The accelerated decay rate in the nonlinear case leads to an over-representation of growing individuals in the whole population for small reserve sizes.

In contrast, the distribution within the growing population $\left(Y_{i} / \sum_{i} Y_{i}\right)$ is conserved 

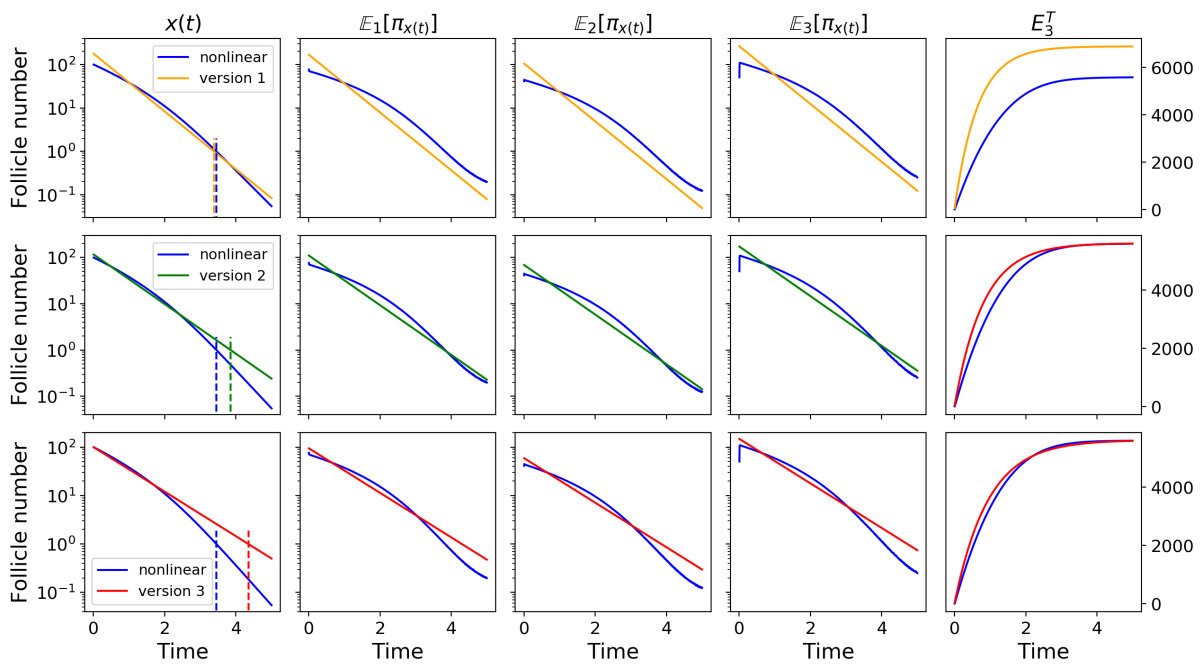

FIG. 3. Comparison between the nonlinear model and three linear formulations fitted on simulated values of $x(t)$ and $Y_{i}(t)_{i=1,2,3}$.

Each column represents the number of follicles in the reserve and fast compartments (from left to right). Each row illustrates the comparison of the nonlinear limit model (blue solid lines), with one of the three fitted linear models. In the first column, the vertical dashed lines represent the extinction time with a threshold level of one remaining follicle. See Table 2 and text body for details on the fitting procedure and parameter values.
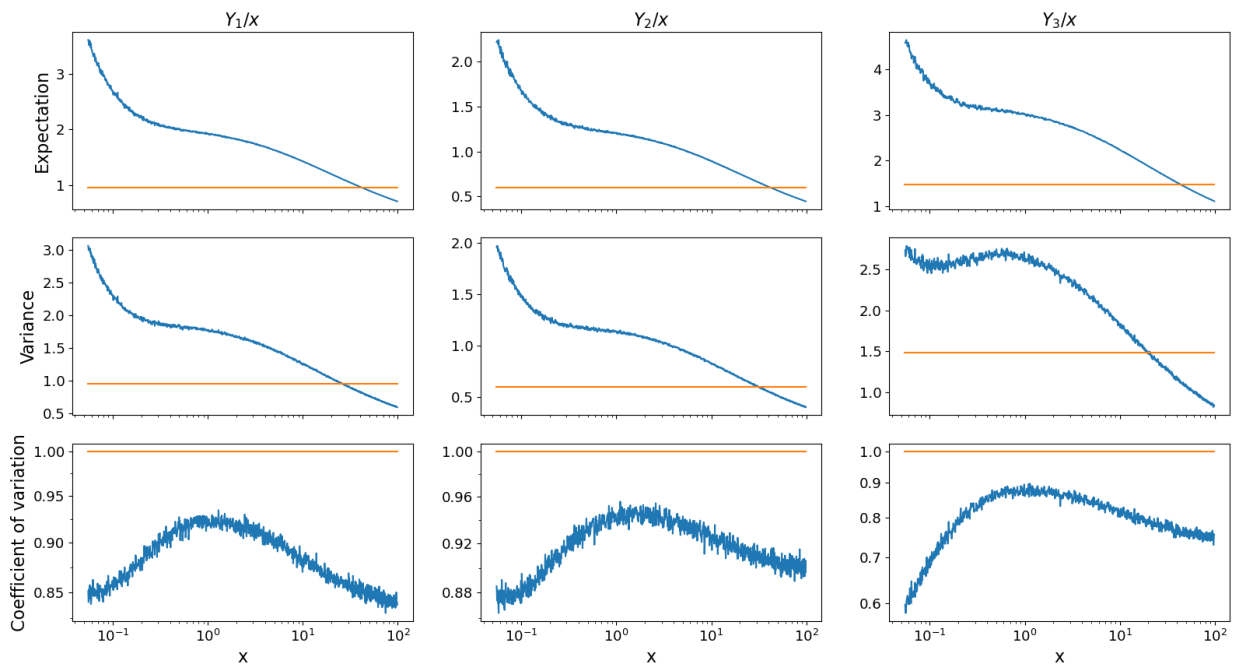

FIG. 4. Expectation, variance and coefficient of variation of the normalized number of growing follicles as a function of $x$ in the linear and nonlinear cases.

Blue lines: nonlinear formulation. Orange lines: linear formulations (version 3 has different values of

$\left(\alpha_{i}\right)_{1 \leq i \leq 3}$ (see Table 2) that are not visually distinguishable from those of versions 1 and 2.). Each column corresponds to the normalized number of growing follicles in the successive compartments (from left to right: $Y_{1} / x, Y_{2} / x$, and $Y_{3} / x$ ). 
through time in both the nonlinear and linear cases (see Figure SM4). This is a consequence of the convergence result on the shape of the growing follicle distribution. Indeed, for any time $t$, the distribution has to be equal to the steady-state distribution associated with the generator, $L_{x(t)}$, which only evolves through the time-dependent input $x(t)$. The growing follicle distribution thus approximately preserves its shape through aging, while decreasing in amplitude, consistently with experimental observations in different species, [21] . Note also that the coefficient of variation is smaller than one in the nonlinear case, suggesting that the feedback mitigates the dispersion in individual numbers.

6. Conclusion. In this study, we have proved rigorously the convergence of a twotimescale stochastic model of population dynamics towards a reduced deterministic limit. The proof follows the general scheme of averaging techniques [14], that we had to adapt to our specific problem. We have obtained compactness and ergodicity properties thanks to a Foster-Lyapunov criterion [16] and coupling techniques. The main idea was to introduce a suitable change of variables to control the time evolution of the population. Due to the unidirectional motion from one compartment to the next, the population in each compartment can hardly be bounded separately, yet the cumulative sums of individual numbers can be controlled by a linear birth-migration process. This idea was used repeatedly to obtain proper control on the moments, the Foster-Lyapunov criterion, and irreducibility. We have also proved that the limit model has a unique solution, and thereby that the full sequence of rescaled processes converges to this solution. In more details, we had to prove that the quasi-stationary distribution of the fast component of the Markov chain is locally Lipschitz with respect to the slow component. To our knowledge, this issue is rarely tackled in details in works dedicated to stochastic averaging. To solve the uniqueness problem, we have introduced a suitable Poisson equation, a tool used in the field of sensitivity analysis. Finally, we have designed a numerical scheme dedicated to the limit model, and based on the nested algorithm [23], widely used in slow/fast problems in chemical kinetics.

From a biomathematical viewpoint, our study can be useful for compartmental systems or birth-death processes with unidirectional migration where the first compartment is much more crowded than the next ones, and has a slow dynamics. It is the case of developmental processes in which individuals in the most immature stage are both more abundant and subject to a slower dynamics than more mature individuals. Such processes are typically undergone in zoological groups spawning eggs and undergoing a metamorphosis process. Amongst these groups, some insect species have been the focus of population dynamics models (see for instance [19]), due to their role as disease vector or crop pest. The slow-fast hypothesis is natural in this context, and has even already been used to study a deterministic model of mosquito populations. In [20], the authors assume that "the egg stock is large, and its dynamics slow compared with the larvae stock". In addition, they introduce a feedback of larvae onto the hatching term, so that, similarly as in our framework, the dynamics of the immature, slow stage is nonlinear and modulated by a faster stage.

From the application viewpoint, our nonlinear formulation generalizes previous attempts to model the dynamics of the ovarian follicle population along life. The limit model approach is based on a multiple timescale hypothesis. This hypothesis can be grounded on quantitative information to set the order of magnitude of the number of quiescent follicles, and of the activation rate. When monitoring the ovarian reserve, hence the number of ovarian follicles a woman (or female mammal) is endowed with at a given age, one usually separates the quiescent follicles from the growing ones. At 
birth, there are only quiescent follicles and the size of the reserve is approximately $510^{5}$ (see [9] for a comparison between the human species and mouse). The total number of follicles continuously decreases until the time of menopause, tens of years later, when the follicle reserve is close to $10^{3}$ follicles. Moreover, while it takes in average fifty years to exhaust the pool of quiescent follicles, the whole growing sequence spans approximately three to four months, as established from cell kinetics studies [12] or grafts of ovarian cortex [4].

Linear population models were considered in a series of articles from MJ Faddy and co-authors, in mice and women (see e.g. [7] and [8]). Time-dependent coefficients were yet needed to achieve the parameter inference from follicle number data, revealing the underlying non-stationarity of the dynamics. Noteworthy, the parameter estimation from data-fitting leads to a much slower rate of activation of the first follicle class than the growth rate of the subsequent classes (corresponding to a small espilon value around $10^{-1} / 10^{-2}$ according to the class number). The results provided in subsection 5.5 clearly illustrate that the nonlinear feedback terms impact the decay rate of the follicle reserve, which speeds up when the growing population diminishes, in consistency with the conclusions of statistical regression models (see e.g. the comprehensive studies [3, 22]). Many interesting and challenging questions remain to be investigated from a physiological and mathematical perspective. We intend to tackle first the issue of solving inverse problems fuelled by data on age-dependent follicle numbers in different species, and to reproduce experimental and physiological situations in which the reserve exhaustion occurs prematurely.

\section{REFERENCES}

[1] C. Bonnet, K. Chahour, F. Clément, M. Postel, and R. Yvinec, Multiscale population dynamics in reproductive biology: Singular perturbation reduction in deterministic and stochastic models, ESAIM Proc. Surveys, 67 (2020), pp. 72-99.

[2] F. Clément and D. Monniaux, Mathematical modeling of ovarian follicle development: A population dynamics viewpoint, Curr. Opin. Endocr. Metab. Res., 18 (2021), pp. 54-61.

[3] J. Coxworth And K. HaWkes, Ovarian follicle loss in humans and mice: Lessons from statistical model comparison, Hum. Reprod., 25 (2010), pp. 1796-1805.

[4] J. Donnez And M.-M. Dolmans, Transplantation of ovarian tissue, Best Pract. Res. Clin. Obstet. Gynaecol., 28 (2014), pp. 1188-1197.

[5] P. Dürrenberger, A. Gupta, and M. Khammash, A finite state projection method for steadystate sensitivity analysis of stochastic reaction networks, Int. J. Chem. Phys., 150 (2019), p. 134101.

[6] S. N. Ethier And T. G. Kurtz, Markov processes: Characterization and convergence, vol. 282 of Wiley Ser. Probab. Stat., Wiley Interscience, 2009.

[7] M. FAdDy And R. Gosden, Physiology: A mathematical model of follicle dynamics in the human ovary, Hum. Reprod., 10 (1995), pp. 770-775.

[8] M. FADDy, E. Jones, AND R. EdwARDs, An analytical model for ovarian follicle dynamics, J. Exp. Zool., 197 (1976), pp. 173-185.

[9] J. Findlay, K. Hutt, M. Hickey, and R. Anderson, How is the number of primordial follicles in the ovarian reserve established, Biol. Reprod., 93 (2015), pp. 111, 1-7, https: //doi.org/10.1095/biolreprod.115.133652.

[10] D. T. GILlESPIE, A general method for numerically simulating the stochastic time evolution of coupled chemical reactions, J. Comput. Phys., 22 (1976), pp. 403-434.

[11] P. W. Glynn And S. P. Meyn, A Liapounov bound for solutions of the Poisson equation, Ann. Probab., 24 (1996), pp. 916-931.

[12] A. Gougeon, Regulation of ovarian follicular development in primates: facts and hypotheses, Endocr. Rev., 11 (1996), pp. 121-155.

[13] H.-W. KAng And T. G. KurTz, Separation of time-scales and model reduction for stochastic reaction networks, Ann. Appl. Probab., 23 (2013), pp. 529-583.

[14] T. G. KuRTz, Averaging for martingale problems and stochastic approximation, in Applied Stochastic Analysis, I. Karatzas and D. Ocone, eds., vol. 177, Springer, 1992, pp. 186-209. 
[15] T. LindVALL, Lectures on the coupling method, Courier Corporation, 2002.

[16] S. P. MEyn AND R. L. TwEedIE, Stability of markovian processes III: Foster-Lyapunov criteria for continuous-time processes, Adv. in Appl. Probab., 25 (1993), pp. 518-548.

[17] S. MÉlÉARd And V. BAnsaye, Some stochastic models for structured populations : Scaling limits and long time behavior, vol. 1, Springer, 2015.

[18] M. F. Neuts And F. P. Kelly, Reversibility and stochastic networks, J. Amer. Statist. Assoc., 76 (1981), p. 492.

[19] G. SchaAlJe AND H. VAn DeR VAART, Relationships among recent models for insect population dynamics with variable rates of development, J. Math. Biol., 27 (1989), pp. 399-428.

[20] M. Strugarek, L. Dufour, N. Vauchelet, L. Almeida, B. Perthame, and D. Villela, Oscillatory regimes in a mosquito population model with larval feedback on egg hatching, J. Biol. Dyn., 13 (2019), pp. 269-300.

[21] C. Thibault, M.-C. Levasseur, And H. R.H.F., Reproduction in mammals and man., INRA Editions, 2001.

[22] W. Wallace AND T. Kelsey, Human ovarian reserve from conception to the menopause, PLoS One., 5 (2010), p. e8772.

[23] E. Weinan, D. LiU, AND E. VANDEN-EIJNDEN, Nested stochastic simulation algorithms for chemical kinetic systems with multiple time scales, J. Comput. Phys., 221 (2007), pp. 158180 .

[24] X. Zhu, D. B. Finlay, M. Glass, and S. B. Duffull, Model-free and kinetic modelling approaches for characterising non-equilibrium pharmacological pathway activity: Internalisation of cannabinoid CB1 receptors, Br. J. Pharmacol., 176 (2019), pp. 2593-2607. 


\section{SM: AVERAGING OF A STOCHASTIC SLOW-FAST MODEL FOR POPULATION DYNAMICS: APPLICATION TO THE DEVELOPMENT OF OVARIAN FOLLICLES*}

GUILLAUME BALLIF ${ }^{\dagger}$, FRÉDÉRIQUE CLÉMENT*, AND ROMAIN YVINEC*‡

SM1. Generator of the coupling. In the proof of Lemma 3.3, we couple processes $\left(X^{\varepsilon}, V^{\varepsilon}\right)$ and $\left(M^{\varepsilon}, W^{\varepsilon}\right)$ with the following generator defined for all functions $h \in$ $\mathcal{C}_{c}^{\infty}\left(\mathbb{R}^{+} \times \mathbb{N}^{d}\right)^{2}$

$$
\begin{aligned}
& C^{\varepsilon} h(x, v, m, w)=\frac{r_{0}(v)}{\varepsilon} x(h(x+\varepsilon, v, m+\varepsilon, w)-h(x, v, m, w)) \\
& +\left(\frac{R_{0}}{\varepsilon} m-\frac{r_{0}(v)}{\varepsilon} x\right)^{+}(h(x, v, m+\varepsilon, w)-h(x, v, m, w)) \\
& +\frac{\lambda_{0}(v)}{\varepsilon} x\left(h\left(x-\varepsilon, v+\sum_{i=1}^{d} e_{i}, m, w+\sum_{i=1}^{d} e_{i}\right)-h(x, v, m, w)\right) \\
& +\left(\frac{B_{0}}{\varepsilon} m-\frac{\lambda_{0}(v)}{\varepsilon} x\right)^{+}\left(h\left(x, v, m, w+\sum_{i=1}^{d} e_{i}\right)-h(x, v, m, w)\right) \\
& +\frac{1}{\varepsilon}\left(\mu_{0}(v) x-\alpha_{0} \min (x, m)\right)[h(x-\varepsilon, v, m, w)-h(x, v, m, w)] \\
& +\frac{\alpha_{0}}{\varepsilon} \min (x, m)\left[h\left(x-\varepsilon, v, m-\varepsilon, w+\sum_{i=1}^{d} e_{i}\right)-h(x, v, m, w)\right] \\
& +\frac{\alpha_{0}}{\varepsilon}(m-\min (x, m))\left[h\left(x, v, m-\varepsilon, w+\sum_{i=1}^{d} e_{i}\right)-h(x, v, m, w)\right] \\
& +\sum_{i=1}^{d} \frac{\alpha_{i} u_{i}(v, w)}{\varepsilon}\left[\frac{\mu_{i}(v)}{\mu_{i}(v)+\lambda_{i}(v)} h\left(x, v-\sum_{j \geq i}^{d} e_{j}, m, w-e_{i}\right)+\frac{\lambda_{i}(v)}{\mu_{i}(v)+\lambda_{i}(v)} h\left(x, v-e_{i}, m, w-e_{i}\right)\right. \\
& -h(x, v, m, w)]+\sum_{i=1}^{d} \frac{\alpha_{i}}{\varepsilon}\left(w_{i}-w_{i-1}-u_{i}(v, w)\right)\left[h\left(x, v, m, w-e_{i}\right)-h(x, v, m, w)\right] \\
& +\sum_{i=1}^{d} \frac{1}{\varepsilon} \frac{\mu_{i}(v)}{\mu_{i}(v)+\lambda_{i}(v)}\left[\left(\mu_{i}(v)+\lambda_{i}(v)\right)\left(v_{i}-v_{i-1}\right)-\alpha_{i} u_{i}(v, w)\right]\left[h\left(x, v-\sum_{j \geq i}^{d} e_{j}, m, w\right)-h(x, v, m, w)\right] \\
& +\sum_{i=1}^{d} \frac{1}{\varepsilon} \frac{\lambda_{i}(v)}{\mu_{i}(v)+\lambda_{i}(v)}\left[\left(\mu_{i}(v)+\lambda_{i}(v)\right)\left(v_{i}-v_{i-1}\right)-\alpha_{i} u_{i}(v, w)\right]\left[h\left(x, v-e_{i}, m, w\right)-h(x, v, m, w)\right]
\end{aligned}
$$

with $u_{i}(v, w)=\min \left(v_{i}-v_{i-1}, w_{i}-w_{i-1}\right)$.

We verify that for any $x \leq m$, and any function h that depends only of $(x, v)$, we have $C^{\varepsilon} h(x, v, m, w)=A^{\varepsilon} h(\bar{x}, v)$. Similarly, for any h that depends only of $(m, w)$, $C^{\varepsilon} h(x, v, m, w)=B^{\varepsilon} h(m, w)$.

\footnotetext{
* Submitted to the editors on April 2, 2021.

${ }^{\dagger}$ Université Paris-Saclay, Inria, Inria Saclay-1̂le-de-France, 91120, Palaiseau, France (guillaume.ballif@inria.fr, frederique.clement@inria.fr)

‡PRC, INRAE, CNRS, Université de Tours, 37380 Nouzilly, France (romain.yvinec@inria.fr)
} 
SM2. One additional lemma to upper bound $B^{\varepsilon} V_{p}$ (Lemma 3.4, subsection 3.1).

Lemma SM2.1. For all $i \in \mathbb{N}^{*}$ and $m \in \mathbb{N}$,

- $D_{i}^{+}(m):=(m+1)^{i}-m^{i} \leq 2^{i}\left(m^{i-1}+1\right)$,

- $m D_{i}^{-}(m):=m\left[(m-1)^{i}-m^{i}\right] \leq-m^{i}$.

Proof. For any $m \geq 1$ :

$$
\begin{aligned}
D_{i}^{+}(m) & =\sum_{j=0}^{i}\left(\begin{array}{c}
i \\
j
\end{array}\right) m^{j}-m^{i}=\sum_{j=0}^{i-1}\left(\begin{array}{l}
i \\
j
\end{array}\right) m^{j} \\
& =m^{i-1} \sum_{k=0}^{i-1}\left(\begin{array}{c}
i \\
k+1
\end{array}\right) \frac{1}{m^{k}} \\
& \leq m^{i-1} \sum_{k=0}^{i-1}\left(\begin{array}{c}
i \\
k+1
\end{array}\right) \text { since } m \geq 1 \\
& \leq 2^{i} m^{i-1} \leq 2^{i}\left(m^{i-1}+1\right) .
\end{aligned}
$$

Given that $D_{i}^{+}(0)=1 \leq 2^{i}$, we get the first inequality.

(SM2.1)

$$
\begin{aligned}
m D_{i}^{-}(m) & =\sum_{j=0}^{i}\left(\begin{array}{l}
i \\
j
\end{array}\right)(-1)^{j} m^{i+1-j}-m^{i+1}=\sum_{j=1}^{i}\left(\begin{array}{l}
i \\
j
\end{array}\right)(-1)^{j} m^{i+1-j} \\
& =m^{i} \sum_{j=1}^{i}\left(\begin{array}{l}
i \\
j
\end{array}\right)(-1)^{j} m^{1-j} \\
& =m^{i} \underbrace{\sum_{k=0}^{i-1}\left(\begin{array}{c}
i \\
k+1
\end{array}\right)(-1)^{k+1} \frac{1}{m^{k}}}_{:=f_{i}(m)} .
\end{aligned}
$$

Since $f_{i}$ is continuous on $\left[1, \infty\left[, f_{i}(1)=-1\right.\right.$ and $\lim _{m \rightarrow \infty} f_{i}(m)=-i, f_{i}$ has a maximum $m_{i}$ on $[1, \infty]$ (which can be reached in infinity). More precisely, $m_{i}$ can be reached either in 1 or in $\left.n_{i} \in\right] 1, \infty$ [ satisfying $f_{i}^{\prime}\left(n_{i}\right)=0$.

We prove by induction on $i \geq 1$ that $m_{i}$ is reached in 1 and that $f_{i}(1)=-1$.

- Initialization: $f_{1}(m)=-1 \leq-1$ for any $m \geq 1$.

- Inductive step: Let $i \geq 1$. We assume that $f_{i}$ reaches its maximum -1 in 1 . The derivation of (SM2.1) gives:

$$
\frac{\mathrm{d} D_{i+1}^{-}}{\mathrm{d} m}(m)=m^{i-1}\left[i f_{i+1}(m)+m f_{i+1}^{\prime}(m)\right] .
$$

By definition of $D_{i+1}^{-}$, we have

$$
(i+1) D_{i}^{-}(m)=m^{i-1}\left[i f_{i+1}(m)+m f_{i+1}^{\prime}(m)\right]
$$


If $m_{i}$ is reached on $] 1, \infty\left[, f_{i+1}^{\prime}\left(m_{i+1}\right)=0\right.$, and we have

$$
\begin{aligned}
f_{i+1}\left(m_{i+1}\right) & =\frac{(i+1) D_{i}^{-}\left(m_{i+1}\right)}{i m_{i+1}^{i-1}} \\
& =\frac{(i+1) m_{i+1}^{i}}{i m_{i+1}^{i-1}} f_{i}\left(m_{i+1}\right) \\
& \leq \frac{(i+1) m_{i+1}}{i} f_{i}\left(m_{i}\right) \\
& \leq-\frac{(i+1) m_{i+1}}{i} \\
& <-1
\end{aligned}
$$

Moreover, $f_{i+1}(1)=\sum_{k=0}^{i}\left(\begin{array}{c}i+1 \\ k+1\end{array}\right)(-1)^{k+1}=-1$.

Hence, we have proved that for any $i \geq 1$, and for any $m \geq 1$, $m D_{i}^{-}(m) \leq-m^{i}$, and it is also true for $m=0$. This gives the second inequality and achieves the proof of (SM2.1).

\section{SM3. Additional Figures.}

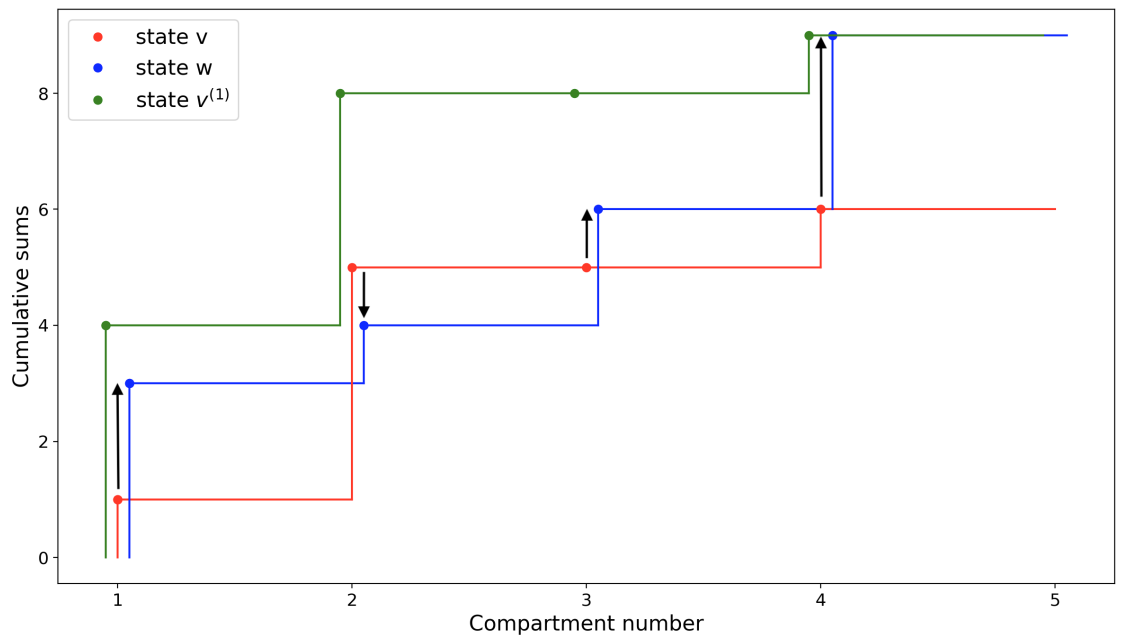

Fig. SM1. Path from $v=(1,5,5,6)$ to $w=(3,4,6,9)$

Cumulative sums of the number of individuals in state $y$ (red solid line), $z$ (blue solid line) and $v^{(1)}$ (green solid line). The black oriented arrows represent $w_{i}-v_{i}$ for each compartment $i$. The first step consists in adding as many individuals as necessary to state $v$ (red lines), so that state $v^{(1)}$ (green lines) overcomes state $w$ (blue lines) in all compartments. The next steps consists in compensating for the numbers of individuals in each compartment, first through migration events and then through death events in the last compartment. 


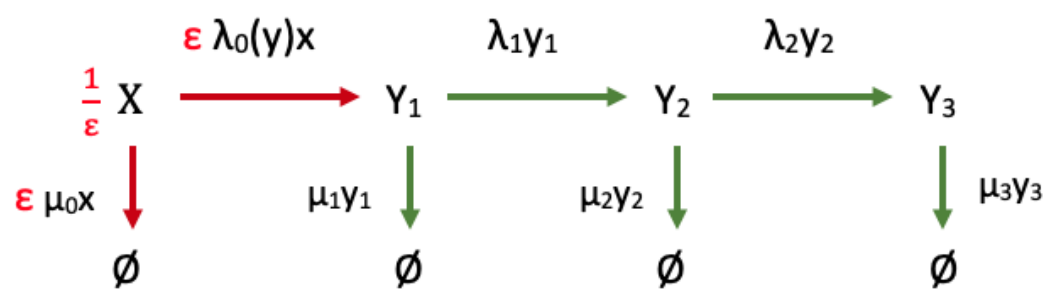

FIG. SM2. Scheme of the model with three compartments, $\left(X^{\varepsilon}, Y_{1}^{\varepsilon}, Y_{2}^{\varepsilon}, Y_{3}^{\varepsilon}\right)$, used to perform the numerical illustrations in section 5 .

All rates are fixed to constant values except $\lambda_{0}$ (see (5.3)).

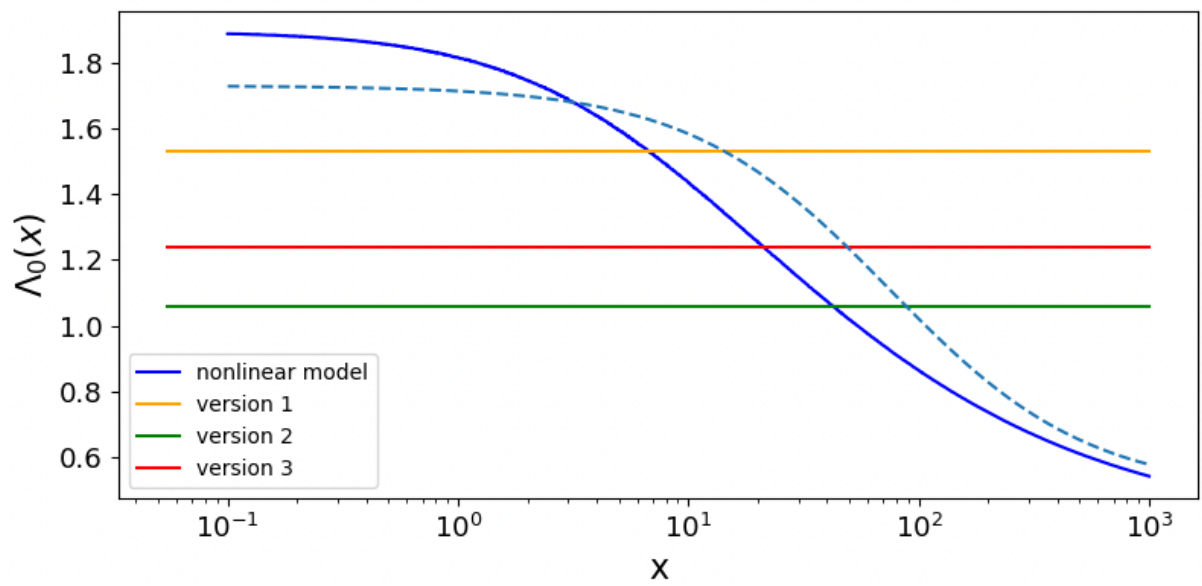

FIG. SM3. $\Lambda_{0}$ (Theorem 2.6) as function of $x$ computed with Algorithm 5.1.

Blue lines : nonlinear model simulated with Algorithm 5.1. Orange (resp. green, red) line: version 1 (resp. version 2, version 3) of the linear formulation. The light-blue dashed line is the graph of the function $x \longrightarrow 0.491+\frac{1.24}{1+0.0134 x}$

Figure SM3 represents the decay rate $\Lambda_{0}$ of the slow compartment in the nonlinear and linear scenarios. From (5.3), in the nonlinear case, this rate evolves between $a+\mu_{0}$ and $a+b+\mu_{0}$. Note that the decay rates inferred from the linear models are all within this range. In the nonlinear scenario, the functional response has a similar shape as the graph of the function $x \longrightarrow a+\frac{b}{1+c x}$ (with $a, b, c$ positive real numbers), which has been proposed as a statistical regression model for the number of quiescent follicles according to age (see subsection 5.5 in the main text). 

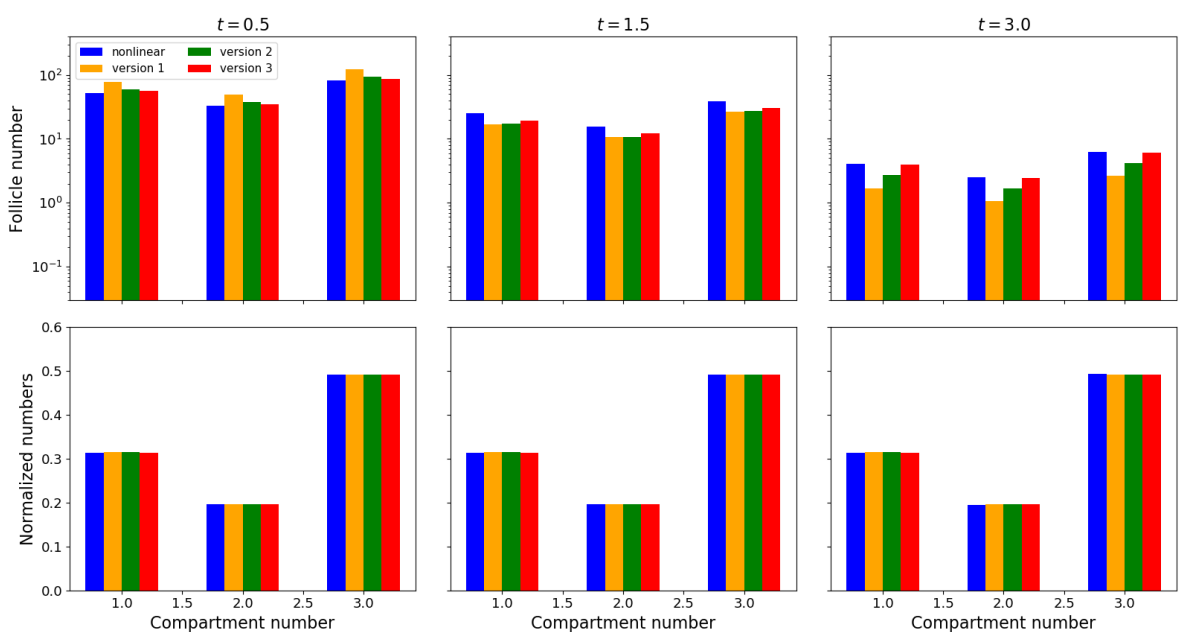

FIG. SM4. Distribution of the growing follicles at different times

Snapshots of the distribution at time $t=0.5$ (left panels), $t=1.5$ (middle panels) and time $t=3$ (right panels). Upper panels : numbers of growing follicles. Lower panels : normalized numbers within the growing population $\left(Y_{i} / \sum_{i} Y_{i}\right)$. Blue bars : nonlinear model. Orange (resp. green, red) bars: version 1 (resp. version 2, version 3) of the linear formulation.

Figure SM4 illustrates the auto-similarity of the quasi-stationary distribution in the fast compartments. Note the almost perfect preservation of shape in the distribution of normalized numbers, and the decrease in the bar amplitudes, as time progresses, in the distribution of non-normalized numbers. 